\title{
Limits of minimal models and continuous orbifolds
}

\author{
Matthias R. Gaberdiel ${ }^{a}$ and Paulina Suchanek ${ }^{a, b}$ \\ ${ }^{a}$ Institut für Theoretische Physik, ETH Zurich, \\ CH-8093 Zürich, Switzerland \\ ${ }^{b}$ Institute for Theoretical Physics, University of Wroctaw, \\ pl. M. Borna 9, 50-204 Wroctaw, Poland \\ E-mail: gaberdiel@itp.phys.ethz.ch, suchanek@itp.phys.ethz.ch
}

\begin{abstract}
The $\lambda=0$ 't Hooft limit of the $2 \mathrm{~d} \mathcal{W}_{N}$ minimal models is shown to be equivalent to the singlet sector of a free boson theory, thus paralleling exactly the structure of the free theory in the Klebanov-Polyakov proposal. In $2 \mathrm{~d}$, the singlet sector does not describe a consistent theory by itself since the corresponding partition function is not modular invariant. However, it can be interpreted as the untwisted sector of a continuous orbifold, and this point of view suggests that it can be made consistent by adding in the appropriate twisted sectors. We show that these twisted sectors account for the 'light states' that were not included in the original 't Hooft limit. We also show that, for the Virasoro minimal models $(N=2)$, the twisted sector of our orbifold agrees precisely with the limit theory of Runkel \& Watts. In particular, this implies that our construction satisfies crossing symmetry.
\end{abstract}

Keywords: AdS-CFT Correspondence, Conformal and W Symmetry

ArXiv EPRINT: 1112.1708 


\section{Contents}

1 Introduction 1

2 Limits of minimal models 3

2.1 The dual gravity perspective 5

$\begin{array}{lll}2.2 & \text { Interpretation as a singlet sector } & 6\end{array}$

3 The continuous orbifold $\quad 7$

$\begin{array}{lll}3.1 & \text { The orbifold projection } & 7\end{array}$

3.2 The twisted sector 8

4 The $c \rightarrow 1$ limit of the Virasoro minimal models $\quad 10$

$\begin{array}{ll}4.1 \text { The identifications } & 10\end{array}$

$\begin{array}{lll}4.2 & \text { Fusion rules } & 11\end{array}$

$\begin{array}{ll}4.3 \text { The full spectrum } & 12\end{array}$

$\begin{array}{ll}4.4 \text { The fractional branes } & 13\end{array}$

$\begin{array}{ll}\text { 4.5 The bulk branes } & 15\end{array}$

5 The twisted sectors from the $\mathcal{W}_{N}$ coset point of view 17

$\begin{array}{llr}6 & \text { Conclusions } & 19\end{array}$

$\begin{array}{ll}\text { A } \quad \text { The case of } \mathrm{SO}(3)=\mathrm{SU}(2) / \mathbb{Z}_{2} & 19\end{array}$

A.1 The untwisted sector 20

A.2 Interpretation in terms of twisted representations 21

$\begin{array}{ll}\text { B Identifying twists with weights } & 23\end{array}$

\section{Introduction}

Simplified versions of the AdS/CFT correspondence hold the promise of offering insights into the mechanism that underly the duality. For example, the large $N$ limit of the CFT at weak coupling [1-4] is believed to be dual to a higher spin theory on the AdS background [5] (see for example [6-9] for reviews). Higher spin theories lie in complexity somewhere between field theories and string theories in that they contain infinitely many fields, but far fewer than a fully fledged string theory. The corresponding duality is therefore much more tractable than the stringy AdS/CFT correspondence, yet contains sufficiently much structure in order to capture many of the essential features.

Some years ago, Klebanov \& Polyakov made a concrete proposal along these lines [10] (for related work see also $[4,11]$ ). They conjectured that Vasiliev's higher spin theory 
on $\mathrm{AdS}_{4}$ is dual to the singlet sector of the $3 \mathrm{~d} \mathrm{O}(N)$ vector model in the large $N$ limit. Recently, impressive evidence in favour of this proposal has been found [12-14], see also [1520] for related work. Last year, a similar duality was proposed in one dimension less [21]: it relates a family of higher spin theories on $\mathrm{AdS}_{3}[22,23]$ to the large $N$ limit of the $\mathcal{W}_{N}$ minimal models in $2 \mathrm{~d}$ (see [24] for a review of $\mathcal{W}$-algebras). This proposal was motivated by the analysis of the asymptotic symmetries of higher spin theories on $\mathrm{AdS}_{3}[25,26]$, following [27], see also [28, 29] for subsequent work. By now it has been shown that the spectra of the two theories agree in the $N \rightarrow \infty$ limit [30] (see also [31]), and correlation functions have been found to match [32-34] (see also [35]). Generalisations for orthogonal groups have been studied [36, 37], and black hole solutions have been analysed [38, 39]; their entropy has (for $\lambda=0,1$ ) been matched to that of the dual CFT [40].

While the proposal of [21] is in many ways the natural lower dimensional analogue of the Klebanov \& Polyakov proposal, the details appear to be somewhat different. For the case of the $\mathrm{O}(N)$ vector model in $3 \mathrm{~d}$, there are two conformal fixed points, the free and the interacting theory, that are believed to be dual to two different higher spin theories on $\mathrm{AdS}_{4}$. In the lower dimensional version, on the other hand, the $\mathcal{W}_{N}$ models possess a line of conformal fixed points in the large $N$ limit that is parametrised by a 't Hooft like coupling $0 \leq \lambda \leq 1$; this is mirrored by the fact that there exists a one-parameter family of higher spin theories on $\mathrm{AdS}_{3}$. It seems natural to think of the theory at $\lambda=0$ as corresponding to the 'free' fixed point, and in this paper we make this correspondence more explicit. The $\lambda=0$ theory corresponds to taking the level $k$ of the $\mathcal{W}_{N}$ minimal model to infinity, before taking $N \rightarrow \infty$. Working at arbitrary finite $N$, we show that the $k \rightarrow \infty$ limit of a $\mathcal{W}_{N}$ minimal model, constructed following [21], can be described as the singlet sector of a free theory (consisting of $N-1$ free bosons). This is therefore the direct analogue of the Klebanov-Polyakov proposal in one higher dimension. For $N=2$, the $k \rightarrow \infty$ limit corresponds to taking the $c \rightarrow 1$ limit of the Virasoro minimal models, and the limit of [21] is analogous to what was considered in [41] (except that we restrict ourselves to a subset of their spectrum for which the partition function converges).

The resulting conformal field theory is well-defined on the sphere, but it is not modular invariant because of the singlet constraint, and hence the resulting conformal field theory is not fully consistent. ${ }^{1}$ However, there is a very natural way in which to repair this: we can think of the singlet condition as an orbifold projection, for which the above singlet sector is the untwisted sector. Then in order to make the theory consistent, all we have to do is to add in the twisted sectors. While this sounds straightforward in principle, there is one somewhat unusual feature: the singlet constraint requires that we orbifold by a continuous compact Lie group (rather than a discrete group), and thus the analysis requires some care. In particular, the twisted sectors are labelled by a continuous parameter (that describes the different conjugacy classes of the orbifold). As we shall see, the ground states of these twisted sectors then have a natural interpretation in terms of the $k \rightarrow \infty$ limit of the $\mathcal{W}_{N}$ minimal models: they describe the 'light states' that were not considered in the limit

\footnotetext{
${ }^{1}$ Note that at finite $N$, the central charge equals $c=N-1$ in this limit, and hence the requirement of modular invariance can be clearly posed.
} 
of [21] since they correspond to states where the size of the Young tableaux scales with $k$ ( or $N$ ). These light states do not contribute in intermediate channels to the correlators of the usual perturbative states from the untwisted sector, because the fusion of states with finitely many Young boxes does not give rise to states where the number of boxes grows with $k$.

Given that our orbifold construction is somewhat unusual - it is the orbifold of $N-1$ free bosons by the continuous group $\mathrm{SU}(N) / \mathbb{Z}_{N}$ - one may worry whether it is in fact consistent. While we cannot prove this in general, we can relate our construction for $N=2$ to a theory that is believed to be consistent. As was mentioned above, the untwisted sector of the $N=2$ orbifold can be thought of as a subsector of the $c \rightarrow 1$ limit of Virasoro minimal models of [41]. It also turns out that the twisted sector has a very natural interpretation: it seems to agree precisely with the alternative $c \rightarrow 1$ limit of the minimal models that was proposed in [42]. In particular, we can show that the spectra coincide, that the fusion rules of [42] are reproduced from our orbifold point of view, and that the boundary conditions from which the construction of [42] originated agree with the usual fractional branes of our orbifold theory. (The non-fractional branes also have a nice interpretation: they correspond precisely to the additional boundary conditions that were later found in [43].) On the other hand, the limit theory of [42] is believed to be consistent — it appears to coincide with the $c \rightarrow 1$ limit of Liouville theory [44] — and it has been checked to satisfy crossing symmetry, which is a highly non-trivial constraint. ${ }^{2}$ Since we can relate our construction to a seemingly consistent conformal field theory, this gives strong evidence in favour of the assertion that our continuous orbifold construction makes sense.

The paper is organised as follows. In section 2 we explain why the $\lambda=0$ theory can be described as the singlet sector of a free theory. In section 3 we show that this projection can be realised as a continuous orbifold, and construct the twisted sector explicitly for the case of $N=2$. In section 4 we explain the close connection between the twisted sector for $N=2$ and the construction of Runkel \& Watts [42]. Section 5 explains the relation between the twisted sector ground states and the 'light states' of the $\mathcal{W}_{N}$ minimal models for large $k$, and section 6 contains our summary and some open problems. There are two appendices where some of the more technical calculations are described.

\section{Limits of minimal models}

The minimal models we are interested in are the $\mathcal{W}_{N}$ coset models

$$
\frac{\mathfrak{s} u(N)_{k} \oplus \mathfrak{s} u(N)_{1}}{\mathfrak{s} u(N)_{k+1}}
$$

that appear in the proposal of [21]. The 't Hooft parameter is defined to be

$$
\lambda=\frac{N}{k+N},
$$

\footnotetext{
${ }^{2}$ One may ask why the twisted sector of an orbifold should by itself satisfy crossing symmetry. The reason is that the contribution from the untwisted sector in intermediate channels is of measure zero and hence does not modify the crossing symmetry analysis.
} 
and the limit of [21] consists of taking $N, k$ to infinity while keeping $\lambda$ fixed. The 'free' theory should correspond to $\lambda=0$, i.e. to the limit where we first take $k \rightarrow \infty$, and then $N \rightarrow \infty$. In this paper we shall mostly study the case of finite $N$; in order to relate our analysis to the $\lambda=0$ case of [21] we should subsequently take $N \rightarrow \infty$.

The central charge of the minimal model (2.1) equals

$$
c=(N-1)\left(1-\frac{N(N+1)}{(N+k)(N+k+1)}\right),
$$

and hence approaches $c \rightarrow N-1$ in the limit $k \rightarrow \infty$. There are different ways in which one may take this limit. In this section we shall define the limit representations by keeping the representation labels of $\mathfrak{s} u(N)$ fixed while taking the limit; this is the analogue of what was done in [21].

In order to understand the resulting representations in detail, it is convenient to describe the coset theory in terms of a Drinfeld-Sokolov (DS) reduction. From this perspective, the representations of the coset theory are labelled by (see for example [24] for an introduction to these matters)

$$
\Lambda=\alpha_{+} \Lambda_{+}+\alpha_{-} \Lambda_{-}
$$

where

$$
\alpha_{+} \alpha_{-}=-1, \quad \alpha_{-}=-\sqrt{k_{\mathrm{DS}}+N}, \quad \alpha_{0}=\alpha_{+}+\alpha_{-},
$$

and $k_{\mathrm{DS}}$ is the level of the DS-reduction; this is related to the level $k$ in the coset description via

$$
\frac{1}{k+N}=\frac{1}{k_{\mathrm{DS}}+N}-1
$$

Furthermore, $\Lambda_{+}$and $\Lambda_{-}$are representations of $\mathfrak{s} u(N)$. In the limit $k \rightarrow \infty$, the level of the DS reduction goes to $k_{\mathrm{DS}} \rightarrow-N+1$, and hence

$$
\alpha_{+} \cong 1, \quad \alpha_{-} \cong-1, \quad \alpha_{0} \cong 0 .
$$

The eigenvalues of the highest weight state $\left(\Lambda_{+} ; \Lambda_{-}\right)$with respect to the zero mode of the (non-primary) spin $s$ fields are (see [24, eq. (6.50)])

$$
\begin{aligned}
u_{s}(\Lambda) & =(-1)^{s-1} \sum_{i_{1}<\cdots<i_{s}} \prod_{j=1}^{s}\left[\left(\Lambda, \varepsilon_{i_{j}}\right)+(s-j) \alpha_{0}\right] \\
& \cong(-1)^{s-1} \sum_{i_{1}<\cdots<i_{s}} \prod_{j=1}^{s}\left(\Lambda_{+}-\Lambda_{-}, \varepsilon_{i_{j}}\right)
\end{aligned}
$$

where $\varepsilon_{i}$ are the weights in the fundamental (vector) representation of $\mathfrak{s u}(N)$, and in going to the second line we have set $\alpha_{0} \cong 0$, as follows from (2.7). Thus in the limit $k \rightarrow \infty$, the coset representation $\left(\Lambda_{+} ; \Lambda_{-}\right)$only depends on $\left(\Lambda_{+}-\Lambda_{-}\right)$; for example, for $N=2$, this is just the familiar statement that, as $k \rightarrow \infty$,

$$
h(r ; s) \simeq \frac{(r-s)^{2}}{4},
$$


where $(r ; s)$ are the usual Kac labels.

The irreducible degenerate $\mathcal{W}_{N}$ representations at $c=N-1$ are thus already accounted for by the representations labelled by $(\Lambda ; 0)$, where $\Lambda$ is an arbitrary weight of $\mathfrak{s u}(N)$; any other degenerate representation, i.e. any representation labelled by $\left(\Lambda_{+} ; \Lambda_{-}\right)$, is $($at $\lambda=0)$ isomorphic to a direct sum of these [30] (see also [41, Remark 4.1.7] for the Virasoro case). In order to determine the actual decomposition, recall that the character of the $\left(\Lambda_{+} ; \Lambda_{-}\right)$ representation equals the branching function of the level $k=1$ affine character with respect to the finite dimensional $\mathfrak{s} u(N)$ representation $\left(\Lambda_{+} \otimes \Lambda_{-}^{*}\right)$, see $[21,24]$. Thus we conclude that the decomposition is

$$
\left(\Lambda_{+} ; \Lambda_{-}\right) \cong \bigoplus_{\Lambda} N_{\Lambda_{+}, \Lambda_{-}^{*}}^{\Lambda}(\Lambda ; 0) \quad \text { for } k \rightarrow \infty,
$$

where $N_{\Lambda_{+}, \Lambda_{-}^{*}}^{\Lambda}$ are the Clebsch-Gordon coefficients

$$
\Lambda_{1} \otimes \Lambda_{2}=\bigoplus_{\Lambda} N_{\Lambda_{1}, \Lambda_{2}}^{\Lambda} \Lambda
$$

Note that this implies in particular that we have the equivalences

$$
(\mathrm{f} ; 0) \cong(0 ; \overline{\mathrm{f}}) \quad \text { and } \quad(\overline{\mathrm{f}} ; 0) \cong(0 ; \mathrm{f}) \quad \text { as } k \rightarrow \infty,
$$

where $\mathrm{f}$ and $\overline{\mathrm{f}}$ denote the fundamental and anti-fundamental representations of $\mathfrak{s u}(N)$, respectively. The natural 'charge-conjugation' theory that contains each of these degenerate representations once is then

$$
\mathcal{H}_{\mathrm{U}}=\bigoplus_{\Lambda} \mathcal{H}_{(\Lambda ; 0)} \otimes \overline{\mathcal{H}}_{\left(\Lambda^{*} ; 0\right)},
$$

where the sum runs over all representations of $\mathfrak{s} u(N)$, and $\Lambda^{*}$ is the conjugate representation to $\Lambda$.

\subsection{The dual gravity perspective}

The equivalence of conformal field theory representations described by (2.10) (and in particular by (2.12)) is also mirrored in the dual higher spin gravity theory, at least if we subsequently take $N \rightarrow \infty$. Recall from [21] that the two complex scalar fields labelled by $[(f ; 0),(\bar{f} ; 0)]$ and $[(0 ; f),(0 ; \bar{f})]$ always have the same mass, but satisfy in general different boundary conditions since the conformal weights of the corresponding boundary fields are

$$
h(\mathrm{f} ; 0)=h(\overline{\mathrm{f}} ; 0)=\frac{1}{2}(1+\lambda), \quad h(0 ; \mathrm{f})=h(0 ; \overline{\mathrm{f}})=\frac{1}{2}(1-\lambda) .
$$

In our limit we have $\lambda=0$, and hence the two boundary conditions coincide. Thus the two complex scalar fields are indistinguishable, i.e. they should describe the 'same' field. It is then natural to consider the subtheory that only contains one of the two complex scalar fields; this is similar to what was proposed (albeit for general $\lambda$ ) in [32]. The dual CFT then only has one set of representations, say those of the form $(\Lambda ; 0)$; its spectrum is thus precisely equal to that in eq. (2.13). 


\subsection{Interpretation as a singlet sector}

Next we want to show that (2.13) actually has a very natural interpretation as the singlet sector of a theory of $(N-1)$ free bosons. In order to see this, recall that the $\mathfrak{s} u(N)$ level $k=1$ theory can be written in terms of $(N-1)$ free bosons compactified on the $\mathfrak{s} u(N)$ lattice. Written in terms of the affine level one representations, the free theory has thus the form

$$
\mathcal{H}_{\text {free }}=\bigoplus_{\mu \in P_{1}^{+}} \mathcal{H}_{\mu}^{\hat{\mathfrak{s u}}} \otimes \overline{\mathcal{H}}_{\mu^{*}}^{\hat{\mathfrak{s}}},
$$

where $\mathcal{H}_{\mu}^{\hat{\mathfrak{s u}}}$ denotes the affine representation labelled by $\mu$, and the sum runs over all integrable level one representations, i.e. those representations where the sum of the Dynkin labels is at most one. Here $\mu^{*}$ is again the conjugate representation to $\mu$.

The $\mathcal{W}_{N}$ algebra at $c=N-1$ can be identified with the Casimir subalgebra of the level one affine algebra [24], i.e. $\mathcal{W}_{N}$ is the commutant of the zero mode algebra $\mathfrak{s u}(N)$ in the vertex operator algebra based on $\hat{\mathfrak{s u}}(N)_{1}$. Thus any representation $\mathcal{H}_{\mu}^{\hat{\mathfrak{s u}}}$ can be decomposed into representations of $\mathfrak{s} u(N) \oplus \mathcal{W}_{N}$,

$$
\mathcal{H}_{\mu}^{\hat{s u}}=\bigoplus_{\Lambda} \Lambda \otimes \mathcal{H}_{(\Lambda ; 0)},
$$

and the usual Howe-type duality arguments (see e.g. [45] for the basic idea) imply that the multiplicity space with which $\Lambda$ appears in $\mathcal{H}_{\mu}^{\hat{\mathfrak{s u}}}$ is an irreducible representation of $\mathcal{W}_{N}$; by comparing the character (see above), it is then clear that the relevant representation must be the one labelled by $(\Lambda ; 0)$. Note that $\Lambda$ runs over all representations of the (finite) Lie algebra for which the center acts as in $\mu$, i.e. for which $\Lambda-\mu$ lies in the root lattice.

Combining (2.15) and (2.16) we now conclude that the free theory has the structure

$$
\mathcal{H}_{\text {free }}=\bigoplus_{\Lambda_{1}, \Lambda_{2}}^{\prime}\left(\Lambda_{1} \otimes \Lambda_{2}^{*}\right) \otimes\left(\mathcal{H}_{\left(\Lambda_{1} ; 0\right)} \otimes \overline{\mathcal{H}}_{\left(\Lambda_{2}^{*} ; 0\right)}\right),
$$

where the sum runs over all representations $\Lambda_{j}$ of $\mathfrak{s} u(N)$, with the only constraint that $\Lambda_{1}-$ $\Lambda_{2}$ lies in the root lattice - this is indicated by the prime. Here the space is decomposed with respect to $\mathfrak{s} u(N) \oplus \mathcal{W}_{N}$, both for left- and right-movers.

It is now immediate that the representation space in (2.13) equals precisely

$$
\mathcal{H}_{\mathrm{U}}=\mathcal{H}_{\text {free }}^{(0)}
$$

where the index (0) means that we restrict ourselves to the subspace of $\mathcal{H}_{\text {free }}$ for which the zero mode action $J_{0}^{a}+\bar{J}_{0}^{a}$ is trivial, i.e. to the states that are singlets under the diagonal action of the left- and right-moving zero mode. Indeed, requiring this singlet condition simply means that we restrict each tensor product $\left(\Lambda_{1} \otimes \Lambda_{2}^{*}\right)$ to the singlet sector; the trivial representation is contained in $\left(\Lambda_{1} \otimes \Lambda_{2}^{*}\right)$ if and only if $\Lambda_{1} \cong \Lambda_{2}$, and if this is the case, it appears with multiplicity one. Thus (2.18) follows from the comparison with (2.13). If we specialise to the case $N=2$, the spectrum of $\mathcal{H}_{\mathrm{U}}$ is a subsector of the spectrum proposed in [41]. 


\section{The continuous orbifold}

The above singlet condition is very reminiscent of what was proposed by Klebanov \& Polyakov in the corresponding $3 \mathrm{~d}$ situation [10]. In the present context, we know that, by itself, the singlet sector is not a consistent conformal field theory since the partition function of $\mathcal{H}_{\mathrm{U}}$ is not modular invariant. However, there is a natural way to complete the above theory to a consistent conformal field theory: we can think of the singlet constraint as the effect of an orbifold projection, and then the completion just consists of adding in the appropriate twisted sectors. There is, however, one subtlety here: the relevant orbifold group is a compact Lie group (rather than a finite discrete group), and hence the analysis requires some care. On the other hand, since compact Lie groups behave in many respects very similar to finite discrete groups, it should not be too surprising that a construction along these lines is possible.

\subsection{The orbifold projection}

The simplest way to describe the singlet condition is via the projection operator

$$
P=\frac{1}{|G|} \int_{G} d \mu(g) g
$$

where $|G|$ is the total volume of $G$ as measured with respect to the Haar measure $d \mu(g)$. The following discussion will be described for an arbitrary Lie group $G$; eventually we shall apply this to the case where the Lie group is $G=\mathrm{SU}(N) / \mathbb{Z}_{N}$, and even more specifically to $G=\mathrm{SU}(2) / \mathbb{Z}_{2} \cong \mathrm{SO}(3) .{ }^{3}$ The partition function from the untwisted sector is then

$$
\begin{aligned}
Z_{\mathrm{U}} & =\frac{1}{|G|} \int_{G} d \mu(g) \operatorname{Tr} \mathcal{H}_{\text {free }}\left(g q^{L_{0}-\frac{c}{24}} \bar{q}^{\bar{L}_{0}-\frac{c}{24}}\right) \\
& =\frac{1}{|G|} \int_{\mathbb{T} / \mathcal{W}} d \hat{\mu}(h) \operatorname{Tr}_{\mathcal{H}_{\text {free }}}\left(h q^{L_{0}-\frac{c}{24}} \bar{q}^{\bar{L}_{0}-\frac{c}{24}}\right),
\end{aligned}
$$

where $h$ is an element in the Cartan torus $\mathbb{T}$, and $\mathcal{W}$ is the Weyl group of $G$. Here we have used that any group element $g \in G$ is conjugate to some element in $\mathbb{T} / \mathcal{W}$, as well as the fact that the trace only depends on the conjugacy class $\operatorname{Con}_{g}$ of $g$. Finally, $d \hat{\mu}(h)$ is the measure

$$
d \hat{\mu}(h)=\operatorname{vol}\left(\operatorname{Con}_{h}\right) d \mu(h) .
$$

The above calculation is illustrated for the case of $\mathrm{SO}(3)$ in appendix A.1, for which $Z_{\mathrm{U}}$ turns out to equal, see eq. (A.11)

$$
Z_{\mathrm{U}}=\sum_{r=0}^{\infty}\left|\chi_{r}(q)\right|^{2}
$$

with

$$
\chi_{r}(q)=\vartheta_{r}(q)-\vartheta_{r+2}(q), \quad \text { and } \quad \vartheta_{r}(q)=\frac{q^{\frac{r^{2}}{4}}}{\eta(q)} .
$$

\footnotetext{
${ }^{3}$ Note that since the representations in $\mathcal{H}_{\text {free }}$ are all pairs of representations $\left(\Lambda_{1} \otimes \Lambda_{2}^{*}\right)$ for which $\Lambda_{1}-\Lambda_{2}$ lies in the root lattice, the center $\mathbb{Z}_{N}$ of $\mathrm{SU}(N)$ acts trivially, and hence the actual orbifold group is $G=\mathrm{SU}(N) / \mathbb{Z}_{N}$.
} 
Since $\chi_{r}(q)$ is the character of the irreducible $c=1$ Virasoro representation labelled by $(r+1 ; 1)$ whose conformal dimension equals $h=\frac{r^{2}}{4}$ in the limit (see $\left.(2.9)\right), Z_{\mathrm{U}}$ agrees indeed with the partition function of (2.13).

\subsection{The twisted sector}

As is familiar from orbifolds of discrete groups, the untwised sector of an orbifold does not define a consistent conformal field theory by itself since the corresponding partition function is not modular invariant (and hence the theory cannot be consistently defined on higher genus surfaces). In order to make the theory consistent we therefore have to add the twisted sectors.

It follows from general orbifold considerations $[46,47]$ that the twisted sectors are labelled by conjugacy classes of group elements. For the case at hand, the twisted sectors are thus labelled by elements $h \in \mathbb{T} / \mathcal{W}$. Each twisted sector (labelled by $h$ ) then has to be projected onto the states that are invariant under the action of the centraliser of $h$ in $G$,

$$
C_{h}=\{g \in G: h g=g h\} .
$$

For a generic element $h \in \mathbb{T} / \mathcal{W}$, the centraliser $C_{h}$ is just the Cartan torus $C_{h}=\mathbb{T}$. Thus the actual contribution of the $h$-twisted sector equals

$$
Z_{\mathrm{T}(h)}=\frac{1}{|\mathbb{T}|} \int_{\mathbb{T}} d \mu(t) \operatorname{Tr}_{\mathcal{H}_{h}}\left(t q^{L_{0}-\frac{c}{24}} \bar{q}^{\bar{L}_{0}-\frac{c}{24}}\right),
$$

where $\mathcal{H}_{h}$ denotes the states in the $h$-twisted sector.

Let us illustrate this for the example of $\mathrm{SO}(3)$, whose untwisted sector is given in (3.4) and worked out in appendix A.1. Using the parametrisation (A.2) we can label the elements of $\mathbb{T} / \mathcal{W}$ by $h=h(\psi)$, where in $\mathrm{SO}(3)$ we have the identifications $\psi \cong \psi+\pi$ and $\psi \cong \pi-\psi$; denoting the representative of $\psi$ with $0 \leq \psi \leq \frac{\pi}{2}$ by $[\psi]$, the elements of $\mathbb{T} / \mathcal{W}$ can thus be labelled by $\alpha \equiv \frac{[\psi]}{\pi} \in\left[0, \frac{1}{2}\right]$.

The partition function of the $\alpha$-twisted sector is obtained by applying the $S$-modular transformation to the trace of the untwisted sector with the insertion of $h(\psi(\alpha))$, i.e. to the integrand of (A.11)

$$
Z_{\mathrm{U}}^{(\alpha)}(\tau)=\sum_{n, w \in \mathbb{Z}} \vartheta_{n+w}(q) \vartheta_{n-w}(\bar{q}) e^{2 \pi i n \alpha}
$$

The $S$-modular transformation of $\vartheta_{r}(q)$ equals

$$
\vartheta_{r}(\tilde{q})=\frac{1}{\sqrt{2}} \int_{-\infty}^{\infty} d s e^{\pi i r s} \vartheta_{s}(q),
$$

where $\tilde{q}=e^{-2 \pi i / \tau}$, and thus

$$
\begin{aligned}
Z_{\mathrm{U}}^{(\alpha)}\left(-\frac{1}{\tau}\right) & =\frac{1}{2} \sum_{n, w \in \mathbb{Z}} \int_{-\infty}^{\infty} d s \int_{-\infty}^{\infty} d \bar{s} e^{2 \pi i n \alpha} e^{\pi i n(s+\bar{s})} e^{\pi i w(s-\bar{s})} \vartheta_{s}(q) \vartheta_{\bar{s}}(\bar{q}) \\
& =\sum_{n \in \mathbb{Z}} \sum_{m \in \mathbb{Z}} \int_{-\infty}^{\infty} d s e^{2 \pi i n \alpha} e^{2 \pi i n s} \vartheta_{s}(q) \vartheta_{s+2 m}(\bar{q}) \\
& =\sum_{m, l \in \mathbb{Z}} \vartheta_{-\alpha+l}(q) \vartheta_{-\alpha+l+2 m}(\bar{q})=\sum_{m, \bar{m} \in \mathbb{Z}, m-\bar{m} \in 2 \mathbb{Z}} \vartheta_{-\alpha+m}(q) \vartheta_{-\alpha+\bar{m}}(\bar{q}) .
\end{aligned}
$$


In the second and third line we have used the identity

$$
\sum_{w \in \mathbb{Z}} e^{i \pi w(s-\bar{s})}=2 \sum_{m \in \mathbb{Z}} \delta(s-\bar{s}+2 m) .
$$

Finally, the projection onto the invariant states in the $\alpha$-twisted sector then leads to

$$
Z_{\mathrm{T}(\alpha)}=\sum_{m \in \mathbb{Z}} \vartheta_{-\alpha+m}(q) \vartheta_{-\alpha+m}(\bar{q})
$$

since the index $-\alpha+m$ and $-(-\alpha+\bar{m})$ can be identified with the left- and right-moving $\mathrm{U}(1)$ charge, respectively; this can for example be deduced from the description of the twisted sector in terms of twisted representations of the affine algebra $\hat{\mathfrak{s u}}(2)$, see appendix A.2 for details. Alternatively, at least for irrational $\alpha$, this projection can also be obtained by demanding invariance under the $T: \tau \mapsto \tau+1$ transformation.

Integrating over the different twist sectors labelled by $\alpha$, the total contribution of the twisted sector is then

$$
\begin{aligned}
Z_{\mathrm{T}} & =\int_{0}^{\frac{1}{2}} d \alpha \sum_{m \in \mathbb{Z}} \vartheta_{-\alpha+m}(q) \vartheta_{-\alpha+m}(\bar{q}) \\
& =\int_{0}^{\prime \infty} d x \vartheta_{x}(q) \vartheta_{x}(\bar{q}) .
\end{aligned}
$$

Strictly speaking the points with $x \in \mathbb{N}$ are excluded from this integral since $\alpha=0$ corresponds to the untwisted sector; this is indicated by the prime in the integral. Our twisted sector agrees then precisely with the partition function that was considered by Runkel \& Watts [42]. We shall elaborate on the precise relation further in section 4.

From the point of view of our orbifold, (3.13) only describes the contribution of the twisted sector. The total partition function should then be obtained by 'adding' to (3.13) the contribution from the untwisted sector (3.4), which contains the irreducible Virasoro representations with $h=\frac{r^{2}}{4}, r \in \mathbb{N}_{0}$. However, in the context of our continuous orbifold we have to be careful how to define this sum since the untwisted sector can be thought of as a twisted sector in the limit of vanishing twist. This suggests that the natural way to include the untwisted sector contribution is to extend the integral in (3.13) to include also the integer points. There is a further subtlety in that the Virasoro characters for $h=\frac{r^{2}}{4}$ are not just $\vartheta_{r}(q)$, but equal $\chi_{r}(q)=\vartheta_{r}(q)-\vartheta_{r+2}(q)$, see (3.5), because of the null-vector at level $r+1$. However, for the purpose of doing the integral this is immaterial since the integer points $x \in \mathbb{N}_{0}$ are of measure zero. Thus we propose that the full partition function equals

$$
Z_{\text {orb }}=\int_{0}^{\infty} d x \vartheta_{x}(q) \vartheta_{x}(\bar{q})
$$

without any restriction on the integral. This is then modular invariant since it equals precisely one half of the partition function of a single uncompactified free boson

$$
Z_{\mathrm{orb}}=\frac{1}{2 \sqrt{\Im(\tau)} \eta(q) \eta(\bar{q})} .
$$


However, as will become clear below, the orbifold theory only shares the partition function with a free boson theory, but is otherwise very different indeed! This is similar to what happened in the construction of Runkel \& Watts [42].

\section{The $c \rightarrow 1$ limit of the Virasoro minimal models}

In the previous section we have proposed that the $k \rightarrow \infty$ limit of the coset models (2.1) can be described in terms of a continuous orbifold of a free boson theory by the compact Lie group $G=\mathrm{SU}(N) / \mathbb{Z}_{N}$. This orbifold construction is somewhat unconventional since the orbifold group in question is continuous rather than discrete. One may therefore worry whether the resulting theory is indeed consistent. As we have seen above, at least for the case of $N=2$, the partition function of the orbifold theory is in fact modular invariant. In this section we want to give further evidence for the consistency of our orbifold for the case of $N=2$.

As we mentioned before the partition function of the twisted sector of the $N=2$ orbifold theory, see (3.13), agrees with the spectrum of the Runkel \& Watts limit [42] of the Virasoro minimal models. In this section, we will argue that this correspondence goes beyond just the level of the spectrum. In particular, after explaining the dictionary between the two descriptions in section 4.1 (see also section 4.3), we show that the fusion rules of [42] have a very natural interpretation from our orbifold point of view (section 4.2). We shall also construct the boundary conditions of [48] that were the starting point of the Runkel \& Watts analysis as fractional branes of our orbifold (section 4.4). Since the Runkel \& Watts limit is believed to define a consistent theory (that can alternatively be described as the $c \rightarrow 1$ limit of Liouville theory, [43, 44]) this in turn also gives strong support to our proposal that our orbifold construction leads to a consistent conformal field theory.

\subsection{The identifications}

Let us first explain the relationship between the two descriptions in detail. In the analysis of Runkel \& Watts [42], the Virasoro primary fields at $c=1$ are labelled by $x \in \mathbb{R}_{+}-\mathbb{N}_{0}$ with $h_{x}=\frac{x^{2}}{4}$. In terms of our orbifold description, the primary $\phi_{x}$ (as well as its Virasoro descendants) comes from the $\alpha$-twisted sector, where

$$
\alpha=\frac{[\psi]}{\pi}=\left\{\begin{array}{cc}
f_{x} & \text { if } 0<f_{x} \leq \frac{1}{2} \\
1-f_{x} & \text { if } \frac{1}{2} \leq f_{x}<1
\end{array} .\right.
$$

Here $[\psi]$ is the representative of $\psi$ with $0<[\psi] \leq \frac{\pi}{2}$ (see the discussion after eq. (3.7)), and $f_{x}$ is the fractional part of $x$,

$$
x=f_{x}+\lfloor x\rfloor,
$$

where $\lfloor x\rfloor$ is the largest integer less than or equal to $x$, i.e. $f_{x}=x-\lfloor x\rfloor$. Note that a representative for the $\alpha$-twist in (4.1) is the group element $h(\psi)$ with $\psi=\pi x$ in the parametrisation (A.2). With these identifications the spectra of the two descriptions match precisely. Indeed, it follows from (A.35) that the $\alpha$-twisted sector (where $0<\alpha \leq \frac{1}{2}$ ) can 
be decomposed in terms of irreducible Virasoro representations as

$$
\mathcal{H}^{(\alpha)}=\bigoplus_{n \in \mathbb{N}_{0}}\left(\mathcal{H}_{h=\frac{(\alpha+n)^{2}}{4}}^{\mathrm{Vir}} \otimes \overline{\mathcal{H}}_{\bar{h}=\frac{(\alpha+n)^{2}}{4}}^{\mathrm{Vir}} \oplus \mathcal{H}_{h=\frac{(1-\alpha+n)^{2}}{4}}^{\mathrm{Vir}} \otimes \overline{\mathcal{H}}_{\bar{h}=\frac{(1-\alpha+n)^{2}}{4}}^{\mathrm{Vir}}\right) .
$$

This then accounts precisely for all $\phi_{x}$-sectors, given the relation (4.1) above.

\section{2 $\quad$ Fusion rules}

Next we want to study the structure of the operator product expansion. It follows from [42, eq. (9)] that the fusion of $\phi_{x}$ with $\phi_{y}$ only contains $\phi_{z}$ provided that either

$$
\lfloor x\rfloor+\lfloor y\rfloor+\lfloor z\rfloor \text { is even and }\left|f_{x}-f_{y}\right|<f_{z}<\min \left(f_{x}+f_{y}, 2-f_{x}-f_{y}\right)
$$

or

$$
\lfloor x\rfloor+\lfloor y\rfloor+\lfloor z\rfloor \text { is odd and }\left|f_{x}-f_{y}\right|<1-f_{z}<\min \left(f_{x}+f_{y}, 2-f_{x}-f_{y}\right) \text {. }
$$

We now want to explain how to reproduce this constraint from the orbifold point of view. From this perspective, the product of a state in the $\alpha_{x}$-twisted sector with a state in the $\alpha_{y}$-twisted sector can only lead to states in the $\alpha_{z}$-twisted sector provided that there are representatives $g_{x}, g_{y}$ and $g_{z}$ in the corresponding conjugacy classes such that [49-51]

$$
g_{z}=g_{x} \cdot g_{y}
$$

Next we recall from (A.4) and (A.6) that the group elements in the conjugacy class of $\alpha_{x}$ can be taken to have $\chi=\psi_{x}=\pi x$ (with $\theta=\theta_{x}$ and $\phi=\phi_{x}$ arbitrary) in the parametrisation (A.1). The product of two group elements with $\chi=\psi_{x}$ and $\chi=\psi_{y}$ is then a group element with $\chi=\psi_{z}$, where

$$
\cos \psi_{z}=\cos \psi_{x} \cos \psi_{y}-\sin \psi_{x} \sin \psi_{y}\left[\cos \theta_{x} \cos \theta_{y}+\sin \theta_{x} \sin \theta_{y} \cos \left(\phi_{x}-\phi_{y}\right)\right] .
$$

The expression in brackets is bounded by

$$
-1 \leq\left[\cos \theta_{x} \cos \theta_{y}+\sin \theta_{x} \sin \theta_{y} \cos \left(\phi_{x}-\phi_{y}\right)\right] \leq 1
$$

and hence

$$
\min \left(\cos \left(\psi_{x}-\psi_{y}\right), \cos \left(\psi_{x}+\psi_{y}\right)\right) \leq \cos \psi_{z} \leq \max \left(\cos \left(\psi_{x}-\psi_{y}\right), \cos \left(\psi_{x}+\psi_{y}\right)\right) .
$$

The further analysis now depends on the parity of $\lfloor x\rfloor+\lfloor y\rfloor$. If $\lfloor x\rfloor+\lfloor y\rfloor$ is even and working with the representatives $\psi_{x}=\pi x$ and $\psi_{y}=\pi y$, then

$$
\begin{aligned}
& \cos \left(\psi_{x}-\psi_{y}\right)=\cos \left(\left|f_{x}-f_{y}\right| \pi\right) \\
& \cos \left(\psi_{x}+\psi_{y}\right)=\cos \left(\left(f_{x}+f_{y}\right) \pi\right)=\left\{\begin{array}{cl}
\cos \left(\left(f_{x}+f_{y}\right) \pi\right) & \text { if } f_{x}+f_{y} \leq 1 \\
\cos \left(\left(2-f_{x}-f_{y}\right) \pi\right) & \text { if } 1<f_{x}+f_{y}<2
\end{array}\right.
\end{aligned}
$$


where the arguments on the right hand side are all in the interval $[0, \pi]$, for which the cosine is injective. Since we also have with $\psi_{z}=\pi z$

$$
\cos \left(\psi_{z}\right)=\left\{\begin{array}{cl}
\cos \left(f_{z} \pi\right) & \text { if }\lfloor z\rfloor \in 2 \mathbb{N} \\
\cos \left(\left(1-f_{z}\right) \pi\right) & \text { if }\lfloor z\rfloor \in 2 \mathbb{N}+1
\end{array}\right.
$$

(4.9) implies for $\lfloor z\rfloor$ even

$$
\left|f_{x}-f_{y}\right| \leq f_{z} \leq \min \left(f_{x}+f_{y}, 2-f_{x}-f_{y}\right)
$$

while for $\lfloor z\rfloor$ odd we have instead

$$
\left|f_{x}-f_{y}\right| \leq 1-f_{z} \leq \min \left(f_{x}+f_{y}, 2-f_{x}-f_{y}\right) .
$$

This then reproduces precisely (4.4) and (4.5), respectively, except that instead of the strict inequalities ' $<$ ', (4.12) and (4.13) involve the non-strict inequalities ' $\leq$ '; this will be commented on in section 4.3 below. The analysis for odd $\lfloor x\rfloor+\lfloor y\rfloor$ is essentially identical. Now the analogue of (4.10) is

$$
\begin{aligned}
& \cos \left(\psi_{x}-\psi_{y}\right)=\cos \left(\left(1-\left|f_{x}-f_{y}\right|\right) \pi\right) \\
& \cos \left(\psi_{x}+\psi_{y}\right)=\cos \left(\left|1-\left(f_{x}+f_{y}\right)\right| \pi\right),
\end{aligned}
$$

and one obtains (4.13) if $\lfloor z\rfloor$ is even, and (4.12) if $\lfloor z\rfloor$ is odd. This then accounts for the remaining cases of (4.4) and (4.5), again except for replacing strict inequalities by non-strict inequalities.

\subsection{The full spectrum}

Recall that the reduced part of the Roggenkamp \& Wendland [41] spectrum (where we restrict ourselves to the representations of the form $(r ; 1))$ describes precisely the untwisted sector of our orbifold, while the Runkel \& Watts spectrum [42] corresponds to the contribution from the twisted sector. The untwisted sector is crossing symmetric by itself, but does not define a consistent theory since the partition function is not modular invariant. On the other hand, the twisted sector is usually, i.e. for standard discrete orbifolds, not consistent by itself since the OPE of two twisted sector states typically also involves untwisted sector contributions. The situation may be slightly different here, since at least crossing symmetry is already satisfied by the twisted sector itself, and the partition function is (at least formally) modular invariant: in both calculations, the contribution from the untwisted sector is of measure zero and therefore does not modify the answer. However, the orbifold point of view suggests that the theory can be (and probably should be) enlarged to contain both twisted and untwisted sector contributions.

Incidentally, the possibility of extending the theory in this manner was already suggested in [42]. As is explained below eq. (6) of that paper, one can fairly naturally introduce the identity operator (corresponding to $x=0$ ) by the formal limit

$$
\mathbf{1}=\lim _{x \rightarrow 0} \frac{1}{x} \phi_{x}
$$


and they indicate that similar constructions should also work for any other $x \in \mathbb{N}$. In terms of the OPE coefficients, this should then in particular mean that one extends the strict inequalities in the fusion rules (4.4) and (4.5) to non-strict inequalities. The resulting extended limit theory should then agree with our continuous orbifold.

\subsection{The fractional branes}

The limit theory of Runkel \& Watts [42] was constructed so as to be compatible with the boundary conditions that had previously been considered in [48]. These boundary conditions are labelled by $a \in \mathbb{N}$, and the open string spectrum between the two boundary conditions $a$ and $b$ equals

$$
\mathcal{H}_{a b}^{\text {open }}=\bigoplus_{r=|a-b|}^{a+b-2} \mathcal{H}_{h=\frac{r^{2}}{4}}^{\mathrm{Vir}}
$$

where the sum over $r$ runs over every other integer, i.e. $r$ is even or odd depending on the parity of $a+b$. We now want to show that these boundary conditions have a natural interpretation from our continuous orbifold point of view.

In order to describe the boundary conditions of the orbifold theory recall that the conformal branes of the 'mother theory', the $\hat{\mathfrak{s u}}(2)$ affine theory at level $k=1$, are labelled by group elements $g \in \mathrm{SU}(2)$ [52], where the corresponding boundary state is characterised by the gluing condition

$$
\left.\left.\left(J_{n}^{a}-g \bar{J}_{-n}^{a} g^{-1}\right) \| g\right\rangle\right\rangle=0 .
$$

Geometrically, the brane corresponding to $g$ describes a D0-brane sitting at the point $g$ on the group manifold [53]. Under the diagonal group action of the element $h \in \mathrm{SO}(3) \cong$ $\mathrm{SU}(2) / \mathbb{Z}_{2}$, the above boundary state gets mapped to

$$
\left.\left.h \| g\rangle\rangle=\| h g h^{-1}\right\rangle\right\rangle,
$$

as follows directly from (4.17): indeed, $h \| g\rangle\rangle$ satisfies the gluing condition

$$
\left.\left.\left(\left(h J_{n}^{a} h^{-1}\right)-h g h^{-1}\left(h \bar{J}_{-n}^{a} h^{-1}\right) h g^{-1} h^{-1}\right) h \| g\right\rangle\right\rangle=0,
$$

and if we redefine the basis of the Lie algebra as $\hat{J}_{n}^{a}=h J_{n}^{a} h^{-1}$, and similarly for the right-movers, we reproduce precisely (4.17) with $g$ replaced by $h g h^{-1}$.

The fixed points of this group action are therefore the branes associated to the identity, $g=\mathbf{1}$, and to the non-trivial element of the center, $g=C$. As is familiar from the general construction of D-branes (or boundary conditions) in orbifold theories, see e.g. [54], the corresponding D-brane is then a 'fractional brane' that will also couple to the twisted sectors of the orbifold. The fractional branes are characterised by a (in general projective) representation $R$ of the orbifold group $G$ [55-57]; this determines the open string spectrum between the boundary conditions labelled by $R$ and $S$ as

$$
Z_{R S}(q)=\frac{1}{|G|} \sum_{g \in G} \operatorname{Tr} \mathcal{H}\left(g q^{L_{0}-\frac{c}{24}}\right) \chi_{R}^{*}(g) \chi_{S}(g),
$$


where $\mathcal{H}$ is the open string spectrum of the brane before orbifolding, and $\chi_{R}(g)$ is the group character of $g$ in the representation $R$. Using

$$
\chi_{R}^{*}(g) \chi_{S}(g)=\sum_{Q} N_{S Q}^{R} \chi_{Q}^{*}(g),
$$

where $N_{S Q}{ }^{R}$ are the Clebsch-Gordon coefficients for the decomposition of $R^{*} \otimes S$ into the representations $Q^{*}$, we can rewrite $(4.20)$ as

$$
Z_{R S}(q)=\sum_{Q} N_{S Q}{ }^{R} \frac{1}{|G|} \sum_{g \in G} \operatorname{Tr}_{\mathcal{H}}\left(g q^{L_{0}-\frac{c}{24}}\right) \chi_{Q}^{*}(g) .
$$

Decomposing the open string spectrum $\mathcal{H}$ with respect to the action of $G$ (as was done in $(2.16))$

$$
\mathcal{H}=\bigoplus_{S} S \otimes \mathcal{H}^{(S)}, \quad \text { so that } \quad \operatorname{Tr}_{\mathcal{H}}\left(g q^{L_{0}-\frac{c}{24}}\right)=\sum_{S} \chi_{S}(g) \operatorname{Tr}_{\mathcal{H}^{(S)}}\left(q^{L_{0}-\frac{c}{24}}\right)
$$

and using the usual orthogonality relation of group characters

$$
\frac{1}{|G|} \sum_{g \in G} \chi_{Q}^{*}(g) \chi_{S}(g)=\delta_{Q S}
$$

the open string spectrum in (4.22) consists then precisely of those states $\mathcal{H}^{(Q)}$ in $\mathcal{H}$ that transform in the $Q$-representation of the orbifold group

$$
Z_{R S}(q)=\sum_{Q} N_{S Q}^{R} \operatorname{Tr}_{\mathcal{H}^{(Q)}}\left(q^{L_{0}-\frac{c}{24}}\right) .
$$

Returning to the case at hand, if both branes are associated to the same fixed point, the relative open string before orbifolding is just the vacuum $(j=0)$ representation of the $\hat{\mathfrak{s u}}(2)$ affine theory at level $k=1$; if the two branes are at different fixed points (one at $g=1$, the other at $g=C$ ), the open string spectrum between them consists of the $j=\frac{1}{2}$ representation of $\mathfrak{s} \hat{u}(2)$. Under the action of the orbifold group these representations decompose as

$$
\mathcal{H}_{j=0}^{\mathfrak{s u}(2)}=\bigoplus_{l \in \mathbb{N}_{0}} D_{l} \otimes \mathcal{H}_{h=l^{2}}^{\mathrm{Vir}}, \quad \mathcal{H}_{j=\frac{1}{2}}^{\mathfrak{s u}(2)}=\bigoplus_{l \in \mathbb{N}_{0}+\frac{1}{2}} D_{l} \otimes \mathcal{H}_{h=l^{2}}^{\mathrm{Vir}}
$$

where $D_{l}$ is the spin $l$ representation of $G=\mathrm{SU}(2)$. Since the projection (4.22) picks out the states that transform in the $Q$ representation, the requirement that the open string spectrum is non-empty demands that $Q$ is half-integer if the two branes in question sit at different fixed points. Thus there is a selection rule for what representations of the orbifold arise: if the fractional brane sits at $g=\mathbf{1}$, say, then $R$ must be a conventional representation of the orbifold group $\mathrm{SO}(3)$, i.e. have integer spin, while for the brane located at $g=C$, the representation $R$ must be projective, i.e. have half-integer spin. (A natural interpretation of this is to say that the orbifold has 'discrete torsion', and that the representation of the orbifold group at the non-trivial fixed point is therefore projective [55-57].) 
Let us denote by $\| g, R\rangle$ the fractional brane sitting at the fixed point $g$ and being characterised by the representation $R$. Then we propose that the branes of [48] are to be identified with the fractional branes in our orbifold as

$$
(a) \Longleftrightarrow\left\{\begin{array}{ll}
\left.\left.\| \mathbf{1}, D_{l(a)}\right\rangle\right\rangle & a \in 2 \mathbb{N}-1 \\
\left.\left.\| C, D_{l(a)}\right)\right\rangle & a \in 2 \mathbb{N},
\end{array} \quad \text { where } \quad l(a)=\frac{a-1}{2} .\right.
$$

With this identification the relative open string spectrum reproduces precisely (4.16). Indeed, the above arguments imply that the projection picks out those Virasoro representations from (4.26) that transform as $a \otimes b$, and this is precisely what (4.16) amounts to.

Incidentally, this identification is also compatible with the bulk boundary couplings. It follows from [42, eq. (14)] that the bulk-boundary coupling of the brane corresponding to $(a)$ equals

$$
B(a ; x)=\sin (\pi a x),
$$

where $x \in \mathbb{R}_{+}$labels the different bulk fields of their analysis. In terms of our orbifold, $B(a ; x)$ should be interpreted as the coefficient with which the above fractional branes couple to the twisted sectors. At least for the case where the representation $R$ is not projective - the situation is more complicated in the projective case [57] - the boundary state of the fractional D-brane sitting at the identity $g=\mathbf{1}$ is schematically (i.e. up to normalisations) of the form

$$
\left.\| \mathbf{1}, R\rangle\rangle=\| \mathbf{1}\rangle\rangle+\sum_{\alpha} \chi_{R}(h(\alpha))|\mathbf{1}\rangle\right\rangle_{\alpha},
$$

where $\| \mathbf{1}\rangle\rangle$ is the boundary state of the original theory as in (4.17), while $|\mathbf{1}\rangle\rangle_{\alpha}$ is the Ishibashi state in the $\alpha$-twisted sector. Here $\chi_{R}(h(\alpha))$ is the character of any representative $h(\alpha)$ in the conjugacy class labelled by $\alpha$, evaluated in the representation $R$. For the case at hand, where we can take $h(\alpha)$ to lie in the Cartan torus and to correspond to the group element (A.2) with $\psi=\pi \alpha$, we have

$$
\chi_{D_{l}}(h(\alpha))=\frac{\sin ((2 l+1) \pi \alpha)}{\sin (\pi \alpha)} .
$$

Since $x$ and $\alpha$ are related as in $(4.1)$, and since $(2 l+1)=a$, see $(4.27)$, we have

$$
B(a ; x)=\sin (\pi x) \chi_{D_{l}}(h(\alpha)) .
$$

Thus the bulk-boundary coupling constants agree up to the irrelevant normalisation constant $\sin (\pi x)$ that is independent of the boundary conditions.

\subsection{The bulk branes}

It was observed in [43] that the limit theory of Runkel \& Watts also possesses another class of boundary conditions that are labelled by $s \in \mathbb{R}$. Actually, the self-spectrum of these D-branes only depends on $s \bmod 1$, and it is given by ${ }^{4}$

$$
\mathcal{S}_{s}=\left\{x \in \mathbb{R}_{+}:-\min \left(2 f_{s}, 2-2 f_{s}\right)<x<\min \left(2 f_{s}, 2-2 f_{s}\right) \bmod 2\right\} .
$$

\footnotetext{
${ }^{4}$ Note that the $p$ parameter of [43] is related to the $x$ parameter of [42] as $p=\frac{x}{2}$.
} 
These branes also have a very natural interpretation from our orbifold point of view: in addition to the fractional branes that are associated to the fixed points of (4.18), the orbifold theory also possesses 'bulk branes' that are simply obtained as orbifold invariant superpositions of the branes of the mother theory, i.e. schematically as

$$
\left.\left.\left.\left.\| \psi\rangle\rangle=\int_{\Gamma} d \mu(g)\left(\| g h(\psi) g^{-1}\right\rangle\right\rangle+\| g h(2 \pi-\psi) g^{-1}\right\rangle\right\rangle\right)
$$

where $\Gamma$ is the set of group elements (A.3) parametrised by $\eta$ and $\varphi$, and $d \mu(g)$ is the restriction of the (suitably rescaled) Haar measure to $\Gamma$. Note that the second term in (4.33) arises because conjugation by the Weyl group element $w \in \mathrm{SO}(3)$ maps $\psi$ to $2 \pi-\psi$, see (A.6).

Obviously $\| \psi\rangle\rangle=\| 2 \pi-\psi\rangle\rangle$, and hence the above boundary conditions are labelled by $\psi \in[0, \pi]$. As we shall see below, we can identify $s=\frac{\psi}{\pi}$, but this then only accounts for $s \in[0,1]$. In order to understand the origin of the integer part of $s$, we note that there is another (hidden) variable characterising these boundary conditions: the above branes are not quite the standard bulk branes since each $\left.\| g h(\psi) g^{-1}\right\rangle$ is actually fixed by a one-dimensional subgroup of the orbifold group, namely by $g \mathbb{T} g^{-1}$. Thus we must specify in addition a representation of $\mathbb{T} \cong \mathrm{U}(1)$, i.e. an integer. This integer then extends $s \in[0,1]$ to $s \in \mathbb{R}$. The integer part of $s$ (i.e. this integer) characterises how the boundary conditions (4.33) couple to the twisted sector of the orbifold; however, as will become clear momentarily, it does not play any significant role for the determination of the self-spectrum, and hence we will not attempt to work this out in detail. Note that this mirrors the fact that $\mathcal{S}_{s}$ in $(4.32)$ also only depends on $s \bmod 1$.

In order to determine the self-spectrum of these boundary conditions (and hence reproduce (4.32)) we recall that the open string spectrum between two boundary states $\left.\left.\| g_{1}\right\rangle\right\rangle$ and $\left.\left.\| g_{2}\right\rangle\right\rangle$ is simply equal to the $g_{1}^{-1} g_{2}$ twisted vacuum representation of $\hat{\mathfrak{s u}}(2)_{1}$, see e.g. [52]. From the point of view of the Virasoro representation theory, the relevant open string spectrum is thus

$$
\mathcal{H}_{0}^{(\beta)}=\bigoplus_{m \in \mathbb{Z}} \mathcal{H}_{h=\frac{(2 m-\beta)^{2}}{4}}^{\mathrm{Vir}}
$$

where $\beta$ is determined by the condition that

$$
g_{1}^{-1} g_{2}=g h(\pi \beta) g^{-1}
$$

for some $g$. (This just means that $h(\pi \beta)$ is the element in the Cartan torus that is conjugate to $g_{1}^{-1} g_{2}$.)

It is now immediate how to determine the open string spectrum of (4.33): the self spectrum of $\| \psi=\pi s\rangle\rangle$ consists of the $\beta$-twisted vacuum representation, where $\beta$ is defined by (4.35), and $g_{1}$ is either conjugate to $h(\pi s)$ or $h\left(\pi(2-s)\right.$ ), and likewise for $g_{2}$. In addition, if both $g_{1}$ and $g_{2}$ are invariant under the same $\mathrm{U}(1)$ subgroup of $\mathrm{SU}(2)$, the relevant open string spectrum must be projected onto the zero U(1) charge sector. ${ }^{5}$ However, this

\footnotetext{
${ }^{5}$ If the two branes have parameters $s_{1}$ and $s_{2}$ with $s_{1}-s_{2} \in \mathbb{Z}$, the open string spectrum must be projected onto the states with $\mathrm{U}(1)$ charge $s_{1}-s_{2}$.
} 
projection only applies to a set of measure zero since generically $g_{1} \mathbb{T} g_{1}^{-1}$ and $g_{2} \mathbb{T} g_{2}^{-1}$ do not coincide. For the purpose of finding the continuous part of the spectrum we can therefore ignore this $\mathrm{U}(1)$ projection.

In order to work out the resulting open string spectrum explicitly, we can follow the same arguments as in section 4.2, see in particular eq. (4.7), to conclude that $\beta$ must satisfy

$$
\cos \left(2 f_{s} \pi\right) \leq \cos (\pi \beta) \leq 1
$$

(This is the condition irrespective of whether $g_{1}$ and $g_{2}$ are conjugate to $h(\pi s)$ or $h(\pi(2-$ s)).) Thus we conclude that

$$
-\min \left(2 f_{s}, 2-2 f_{s}\right) \leq \beta \leq \min \left(2 f_{s}, 2-2 f_{s}\right) \quad \bmod 2 .
$$

Together with (4.34) this then reproduces precisely (4.32), apart from the by now familiar difference between strict inequalities and non-strict inequalities.

\section{The twisted sectors from the $\mathcal{W}_{N}$ coset point of view}

In the previous section we have shown that for the case of $N=2$, our orbifold theory is very closely related to the construction of Runkel \& Watts [42]. In this section we want to return to the general case. We want to explain that the ground states of the twisted sectors are directly related to the 'light' states of the $\mathcal{W}_{N}$ minimal models in the $k \rightarrow \infty$ limit $[21,30]$.

As was explained in detail in section 4.1, for the case of $N=2$ the label of the twist sectors $\alpha \in\left[0, \frac{1}{2}\right]$ is related to the parameter $x$ of Runkel \& Watts [42] as in (4.1); in particular, for $x \in\left[0, \frac{1}{2}\right]$ we simply have $\alpha=x$. On the other hand, it is implicit from the analysis of Runkel \& Watts [42] (see also [43]) that we can think of the fields labelled by $x \in\left[0, \frac{1}{2}\right]$ as the limit of the $(r ; r)$ fields for which $r$ is not kept constant as $p=k+2$ is taken to infinity, but rather scales as $r \sim \alpha p$. Indeed, the conformal dimension of the $(r ; s)$ representation has the expansion

$$
h(r ; s) \simeq \frac{(r-s)^{2}}{4}+\frac{r^{2}-s^{2}}{4 p}+\frac{s^{2}-1}{4 p^{2}}+\mathcal{O}\left(\frac{1}{p^{3}}\right) .
$$

Thus we have for $r=s=\alpha p$

$$
\left.h(r ; r)\right|_{r=\alpha p} \simeq \frac{\alpha^{2} p^{2}-1}{4 p^{2}} \simeq \frac{\alpha^{2}}{4} .
$$

We therefore conclude that we can identify the ground states of the twisted sectors of our continuous orbifold with the 'light' states of the $c \rightarrow 1$ limit of the Virasoro minimal models.

We now want to argue that a similar relation holds for the $\mathcal{W}_{N}$ case (see also [58] where some aspects of the Runkel \& Watts analysis have been generalised to the $\mathcal{W}_{N}$ case). Recall from $[21,30]$ that the light states of the $k \rightarrow \infty$ limit of the $\mathcal{W}_{N}$ coset theory arise for $\Lambda_{+}=\Lambda_{-}=\Lambda$, for which the conformal dimension is of the form

$$
h(\Lambda ; \Lambda)=\frac{1}{2 p(p+1)}(\Lambda, \Lambda+2 \rho),
$$


where $p=k+N, \rho$ is the Weyl vector of $\mathfrak{s u}(N)$, and $(\cdot, \cdot)$ denotes the usual inner product on the weight space. Writing $\Lambda$ in terms of Dynkin labels, $\Lambda=\left[\Lambda_{1}, \ldots, \Lambda_{N-1}\right]$, we have (see e.g. appendix B.1 of [21])

$$
\frac{1}{2 p(p+1)}(\Lambda, 2 \rho)=\frac{1}{p(p+1)} \sum_{j=1}^{N-1} \Lambda_{j} \frac{j(N-j)}{2} \leq \frac{D(N)}{p(p+1)} \sum_{j=1}^{N-1} \Lambda_{j} \leq \frac{D(N) k}{p(p+1)}
$$

where $D(N)$ is some $N$-dependent constant, and we have used that $\Lambda$ is an integrable weight at level $k$ and hence satisfies $\sum_{j} \Lambda_{j} \leq k$. As we take $k \rightarrow \infty$ for fixed $N$, the right hand side goes to zero. Thus in this limit we have (compare also [58])

$$
h(\Lambda ; \Lambda) \simeq \frac{1}{2 p(p+1)}(\Lambda, \Lambda) \simeq \frac{1}{2}(\tilde{\Lambda}, \tilde{\Lambda}), \quad \text { with } \quad \tilde{\Lambda}=\frac{1}{p} \Lambda .
$$

The 'light states' are therefore obtained by scaling the representations $\Lambda^{(p)}$ with $p$ such that $\tilde{\Lambda}=\frac{1}{p} \Lambda^{(p)}$ approaches a constant vector. Since each $\Lambda^{(p)}$ must be an integrable weight at level $k=p-N$, it follows that $\tilde{\Lambda}$ must satisfy

$$
\sum_{j=1}^{N-1} \tilde{\Lambda}_{j} \leq 1
$$

where $\tilde{\Lambda}=\left[\tilde{\Lambda}_{1}, \ldots, \tilde{\Lambda}_{N-1}\right]$ in the usual Dynkin basis. Furthermore we have $\tilde{\Lambda}_{j} \geq 0$.

As in the Virasoro case above, we now want to identify (a subset of) these $\tilde{\Lambda}$ with the different twists of our continuous orbifold. Recall from the discussion of section 3.2 that the different twist sectors are labelled by $\alpha$, where $\alpha$ parametrises the elements in $\mathbb{T} / \mathcal{W}$, with $\mathbb{T}$ the Cartan torus and $\mathcal{W}$ the Weyl group of $\mathrm{SU}(N) / \mathbb{Z}_{N}$. Using the description in terms of twisted representations as in section A.2, it follows that the conformal dimension of the $\alpha$-twisted sector ground state equals (see e.g. [59, eq. (4.7)])

$$
h(\alpha)=\frac{1}{2}(\alpha, \alpha),
$$

where $\alpha$ is now thought of as a weight, with $(\cdot, \cdot)$ the natural inner product on the weight space. The comparison with (5.5) thus suggests that we should identify

$$
\tilde{\Lambda}=\alpha .
$$

As is shown in appendix B, the weights $\tilde{\Lambda}$ satisfying $\tilde{\Lambda}_{j} \geq 0$ and (5.6) are in one-to-one correspondence with the weights $\alpha$ parametrising the elements in $\hat{\mathbb{T}} / \mathcal{W}$, where $\hat{\mathbb{T}}$ is the Cartan torus of $\mathrm{SU}(N)$. For the actual quotient space $\mathbb{T} / \mathcal{W}$, where $\mathbb{T}$ is the Cartan torus of $\mathrm{SU}(N) / \mathbb{Z}_{N}$, the weights $\alpha$ have in addition to satisfy (B.13) and (B.14), which is the analogue of the constraint $\alpha \leq \frac{1}{2}$ (rather than $\alpha \leq 1$ ) for the case of $\mathrm{SO}(3)=\mathrm{SU}(2) / \mathbb{Z}_{2}$. This therefore demonstrates that the light states of small conformal dimension can be identified with the ground states of the twisted sectors. The remaining light states (as well as some of the states corresponding to the scaled representations with $\Lambda_{+} \neq \Lambda_{-}$) correspond then to descendants in these twisted sectors. 


\section{Conclusions}

In this paper we have shown that the $\lambda=0$ 't Hooft limit of the $\mathcal{W}_{N}$ minimal models [21] can be identified with the singlet sector of a free boson theory. This is the natural analogue of the free fixed point of the $\mathrm{O}(N)$ vector model that appeared in the duality of Klebanov \& Polyakov in one dimension higher [10]. The singlet sector of the free boson theory in 2 dimensions is not a consistent conformal field theory by itself since the corresponding partition function is not modular invariant. However, one can think of it as the untwisted sector of a continuous orbifold. This implies that it can be made consistent by adding in the appropriate twisted sectors. The relevant twisted sectors correspond precisely to the 'light states' of small conformal dimension; they were not included in the limit of [21].

Our orbifold construction is somewhat unusual in that the orbifold group is continuous (and compact) rather than discrete. As a consequence one may be worried about the consistency of the resulting theory. In order to dispel this suspicion we have shown that for $N=2$, i.e. the $c \rightarrow 1$ limit of the Virasoro minimal models, our construction is closely related to the model proposed in [42]. Given that the latter is known to satisfy a number of non-trivial consistency conditions (in particular crossing symmetry), this implies that the same is true for our continuous orbifold, at least for $N=2$. Recently the analysis of [42] was partially generalised to $N>2$ in [58], where it was argued that the limit theory can be identified with a Toda field theory, see also [60]; it would be interesting to check that also these limit theories allow for an orbifold interpretation as argued above.

In the context of the higher spin duality, our analysis gives a nice CFT interpretation to the 'light states' at $\lambda=0$. One may wonder to which extent this description could also work for $\lambda>0$. Obviously, for $\lambda>0$, the theory is no longer free, but it would be interesting to understand whether some aspects of the orbifold description survive when the coupling is switched on. It would also be interesting to understand the relation of these twisted sectors to the recent proposal that the light states correspond to conical surpluses [61].

\section{Acknowledgments}

We thank Stefan Fredenhagen, Rajesh Gopakumar, Tom Hartman, Emil Martinec, Shiraz Minwalla, Mukund Rangamani, Ingo Runkel and Ashoke Sen for useful discussions. The work of PS is supported by a Sciex grant of the CRUS, and the work of MRG is partially supported by a grant from the Swiss National Science Foundation. MRG is grateful to the Aspen Center of Physics for hospitality while some of this work was being carried out.

\section{A The case of $\mathrm{SO}(3)=\mathrm{SU}(2) / \mathbb{Z}_{2}$}

In this appendix we calculate the partition function $Z_{\mathrm{U}}$ (see eq. (3.2)) of the untwisted sector explicitly for the case of $\mathrm{SO}(3)=\mathrm{SU}(2) / \mathbb{Z}_{2}$. We also explain how the corresponding twisted sectors can be described in terms of twisted representations of $\hat{\mathfrak{s u}}(2)$. 


\section{A.1 The untwisted sector}

Let us parametrise an arbitrary group element in $\mathrm{SU}(2)$ as (see e.g. [62, eq. (2.5)])

$$
g(\chi, \theta, \phi)=\left(\begin{array}{cc}
\cos \chi+i \sin \chi \cos \theta & i \sin \chi \sin \theta e^{i \phi} \\
i \sin \chi \sin \theta e^{-i \phi} & \cos \chi-i \sin \chi \cos \theta
\end{array}\right),
$$

where $\chi, \phi \in[0,2 \pi]$, while $\theta \in\left[0, \frac{\pi}{2}\right]$. In order to describe $\mathrm{SO}(3)=\mathrm{SU}(2) / \mathbb{Z}_{2}$, we have to identify $\chi \cong \chi+\pi$, so that for $\mathrm{SO}(3)$ we only have $\chi \in[0, \pi]$. We take the Cartan torus of $\mathrm{SU}(2)$ to consist of the group elements of the form

$$
h(\psi)=\left(\begin{array}{cc}
\cos \psi & i \sin \psi \\
i \sin \psi & \cos \psi
\end{array}\right),
$$

where $\psi \in[0,2 \pi]$; for $\mathrm{SO}(3)$, the Cartan torus $\mathbb{T}$ is then of the same form, except that $\psi \in[0, \pi]$. For

$$
g(\eta, \varphi)=\frac{1}{\sqrt{2}}\left(\begin{array}{cc}
e^{i \eta} & e^{i \varphi} \\
-e^{-i \varphi} & e^{-i \eta}
\end{array}\right)
$$

we find

$$
\begin{aligned}
g(\eta, \varphi) h(\psi) g(\eta, \varphi)^{-1} & =\left(\begin{array}{cc}
\cos \psi+i \sin \psi \cos (\varphi-\eta) & \sin \psi \sin (\varphi-\eta) e^{i(\varphi+\eta)} \\
-\sin \psi \sin (\varphi-\eta) e^{-i(\varphi+\eta)} & \cos \psi-i \sin \psi \cos (\varphi-\eta)
\end{array}\right) \\
& =g\left(\psi, \varphi-\eta, \varphi+\eta-\frac{\pi}{2}\right)
\end{aligned}
$$

in the notation of (A.1). Thus every group element in $\mathrm{SU}(2)$ is in the conjugacy class of an element of the Cartan torus, and similarly for $\mathrm{SO}(3)$.

The Weyl group of $\mathrm{SU}(2)$ is $\mathbb{Z}_{2}$, and it is generated by the group element

$$
w=\left(\begin{array}{rr}
0 & 1 \\
-1 & 0
\end{array}\right)
$$

which maps the Cartan torus under conjugation to itself

$$
h(\psi) \mapsto w h(\psi) w^{-1}=\left(\begin{array}{cc}
\cos \psi & -i \sin \psi \\
-i \sin \psi & \cos \psi
\end{array}\right)=h(2 \pi-\psi) .
$$

For $\mathrm{SO}(3)$, where $\psi \in[0, \pi]$, the Weyl group then identifies $\psi \cong \pi-\psi$. In the following it will be convenient to take $\psi \in \mathbb{R}_{+}$, and to define $[\psi]$ to be the representative of $\psi$ (after using the identifications $\psi \cong \psi+\pi$ and $\psi \cong \pi-\psi$ ) with $0<[\psi] \leq \frac{\pi}{2}$. We shall usually parametrise the set $\mathbb{T} / \mathcal{W}$ instead of $[\psi]$ by $\alpha \equiv \frac{[\psi]}{\pi} \in\left[0, \frac{1}{2}\right]$.

Using the coordinates in (A.1), the Haar measure on $\mathrm{SU}(2)$ takes the form

$$
d \mu=\sin ^{2} \chi \sin \theta d \chi d \theta d \phi,
$$

and thus the volume of $\mathrm{SO}(3)$ is

$$
|\mathrm{SO}(3)|=\int_{0}^{\pi} d \chi \sin ^{2} \chi \int_{0}^{\frac{\pi}{2}} d \theta \sin \theta \int_{0}^{2 \pi} d \phi=\pi^{2}
$$


while the volume of the conjugacy class containing $h(\psi)$ equals

$$
\operatorname{vol}\left(\operatorname{Con}_{h(\psi)}\right)=2 \pi \sin ^{2}(\psi)+2 \pi \sin ^{2}(\pi-\psi)=4 \pi \sin ^{2}(\psi) .
$$

In order to determine the contribution from the untwisted sector recall that the partition function of a free boson at the self-dual radius equals

$$
Z_{\text {free }}=\frac{1}{\eta \bar{\eta}} \sum_{n, w} q^{\frac{(n+w)^{2}}{4}} \bar{q} \frac{(n-w)^{2}}{4},
$$

where $\eta \equiv \eta(\tau)$ is the Dedekind eta function, and $\bar{\eta} \equiv \eta(\bar{\tau})$, with $q=\exp (2 \pi i \tau)$ and $\bar{q}=\exp (-2 \pi i \bar{\tau})$. Imposing the projection of $(3.2)$ then leads to the untwisted sector partition function

$$
\begin{aligned}
Z_{\mathrm{U}} & =\frac{4}{\pi} \int_{0}^{\frac{\pi}{2}} d \psi \sin ^{2}(\psi) \frac{1}{\eta \bar{\eta}} \sum_{n, w \in \mathbb{Z}} q^{\frac{(n+w)^{2}}{4}} \bar{q}^{\frac{(n-w)^{2}}{4}} e^{2 i n \psi} \\
& =\frac{4}{\pi} \int_{0}^{\frac{\pi}{2}} d \psi \sin ^{2}(\psi) \frac{1}{\eta \bar{\eta}} \sum_{n, w \in \mathbb{Z}} q^{\frac{(n+w)^{2}}{4}} \bar{q}^{\frac{(n-w)^{2}}{4}} \cos (2 n \psi) \\
& =\frac{1}{\eta \bar{\eta}}\left(\sum_{w \in \mathbb{Z}} q^{\frac{w^{2}}{4}} \bar{q}^{\frac{w^{2}}{4}}-\frac{1}{2} \sum_{w \in \mathbb{Z}} q^{\frac{w^{2}}{4}} \bar{q} \frac{(w+2)^{2}}{4}-\frac{1}{2} \sum_{w \in \mathbb{Z}} q^{\frac{(w+2)^{2}}{4}} \bar{q} \frac{w^{2}}{4}\right)=\sum_{r=0}^{\infty}\left|\chi_{r}(q)\right|^{2},
\end{aligned}
$$

where $\chi_{r}(q)$ is defined in (3.5), and we have used that

$$
\int_{0}^{\frac{\pi}{2}} d \psi \sin ^{2}(\psi) \cos (2 n \psi)=\left\{\begin{array}{rl}
\frac{\pi}{4} & n=0 \\
-\frac{\pi}{8} & n= \pm 1 \\
0 & n \in \mathbb{Z} \backslash\{0, \pm 1\}
\end{array}\right.
$$

\section{A.2 Interpretation in terms of twisted representations}

The $\alpha$-twisted sector can also be interpreted in terms of twisted representations of the affine $\mathfrak{s u}(2)$ algebra, for a review of twisted representations see e.g. [63, section 3.5]. Recall that the free boson theory (A.10) is actually equivalent to the level one affine $\hat{\mathfrak{s u}}(2)$ theory. The twisted sectors are then described by twisted representations of the affine $\mathfrak{s u}(2)$ theory. Since the twists are inner, the corresponding twisted algebras are all isomorphic to the untwisted algebra.

In order to explain this in more detail, let us fix conventions for the $\hat{\mathfrak{s u}}(2)$ affine algebra at level $k$. In the Cartan-Weyl basis it is generated by the modes

$$
\begin{aligned}
& {\left[J_{m}^{3}, J_{n}^{ \pm}\right]= \pm J_{m+n}^{ \pm}, \quad\left[J_{m}^{3}, J_{n}^{3}\right]=\frac{k}{2} m \delta_{m,-n}} \\
& {\left[J_{m}^{+}, J_{n}^{-}\right]=2 J_{m+n}^{3}+k m \delta_{m,-n} .}
\end{aligned}
$$

In addition we have the Virasoro modes $L_{m}$, whose commutation relations are

$$
\begin{aligned}
{\left[L_{m}, L_{n}\right] } & =(m-n) L_{m+n}+\frac{c}{12} m\left(m^{2}-1\right) \delta_{m,-n} \\
{\left[L_{m}, J_{n}^{a}\right] } & =-n J_{m+n}^{a} .
\end{aligned}
$$


The modes of the $\alpha$-twisted algebra are then of the form $K_{m}^{3}, K_{s}^{ \pm}$, where $m \in \mathbb{Z}$ while the modings of the $K_{s}^{ \pm}$generators are $s \in \mathbb{Z} \pm \alpha$, respectively. Furthermore, we denote by $\hat{L}_{m}$ the Virasoro modes in the twisted representation. These modes satisfy formally the same commutation relations as the $J_{m}^{a}$ and $L_{m}$, i.e.

$$
\begin{aligned}
{\left[K_{m}^{3}, K_{s}^{ \pm}\right] } & = \pm K_{m+s}^{ \pm}, \quad\left[K_{m}^{3}, K_{n}^{3}\right]=\frac{k}{2} m \delta_{m,-n} \\
{\left[K_{r}^{+}, K_{s}^{-}\right] } & =2 K_{r+s}^{3}+k r \delta_{r,-s} \\
{\left[\hat{L}_{m}, \hat{L}_{n}\right] } & =(m-n) \hat{L}_{m+n}+\frac{c}{12} m\left(m^{2}-1\right) \delta_{m,-n} \\
{\left[\hat{L}_{m}, K_{p}^{a}\right] } & =-p K_{m+p}^{a} .
\end{aligned}
$$

The two algebras are isomorphic, the isomorphism being given by

$$
\begin{aligned}
\varphi_{\alpha}\left(J_{m}^{ \pm}\right) & =K_{m \pm \alpha}^{ \pm} \\
\varphi_{\alpha}\left(J_{m}^{3}\right) & =K_{m}^{3}+\frac{\alpha}{2} k \delta_{m, 0} \\
\varphi_{\alpha}\left(L_{m}\right) & =\hat{L}_{m}+\alpha K_{m}^{3}+\frac{k}{4} \alpha^{2} \delta_{m, 0}
\end{aligned}
$$

as one can easily verify explicitly. The inverse map is then simply

$$
\begin{aligned}
& \varphi_{\alpha}^{-1}\left(K_{s}^{ \pm}\right)=J_{s \mp \alpha}^{ \pm} \\
& \varphi_{\alpha}^{-1}\left(K_{m}^{3}\right)=J_{m}^{3}-\frac{\alpha}{2} k \delta_{m, 0} \\
& \varphi_{\alpha}^{-1}\left(\hat{L}_{m}\right)=L_{m}-\alpha J_{m}^{3}+\frac{k}{4} \alpha^{2} \delta_{m, 0} .
\end{aligned}
$$

With these preparations it is now easy to describe the twisted representations. The untwisted highest weight representations are labelled by $j=0, \frac{1}{2}, \ldots, \frac{k}{2}$, and they are generated from a highest weight states satisfying

$$
J_{n}^{a}|j\rangle=0 \quad(n>0), \quad J_{0}^{+}|j\rangle=0, \quad J_{0}^{3}|j\rangle=j|j\rangle, \quad L_{0}|j\rangle=\frac{j(j+1)}{k+2}|j\rangle,
$$

by the action of the negative modes. The representation has a singular vector of the form

$$
\left(J_{-1}^{+}\right)^{k+1-2 j}|j\rangle \cong 0
$$

which generates the full null space. The twisted representation acts on the same vector space, but we describe the action in terms of the $K_{p}^{a}$ and $\hat{L}_{m}$ modes, using $\varphi_{\alpha}^{-1}$. Since $0<\alpha \leq \frac{1}{2}$ - in fact $0<\alpha<1$ would suffice - the ground state $|j\rangle$ is still highest weight with respect to the twisted modes as

$$
\begin{aligned}
& K_{s}^{+}|j\rangle=J_{s-\alpha}^{+}|j\rangle=0 \quad \text { for } \quad s=m+\alpha>0 \\
& K_{s}^{-}|j\rangle=J_{s+\alpha}^{-}|j\rangle=0 \quad \text { for } \quad s=m-\alpha>0 .
\end{aligned}
$$


However, the $K_{0}^{3}$ and $\hat{L}_{0}$ eigenvalues are now shifted as

$$
K_{0}^{3}|j\rangle=\left(j-\frac{k \alpha}{2}\right)|j\rangle, \quad \hat{L}_{0}|j\rangle=\left(\frac{j(j+1)}{k+2}-\alpha j+\frac{\alpha^{2}}{4}\right)|j\rangle .
$$

For the case of $k=1$ (that is of primary interest to us), the possible values of $j$ are $j=0$ and $j=\frac{1}{2}$. Then the corresponding eigenvalues are

$$
\begin{array}{rlrl}
K_{0}^{3}|0\rangle & =-\frac{\alpha}{2}|0\rangle & \hat{L}_{0}|0\rangle & =\frac{\alpha^{2}}{4}|0\rangle \\
K_{0}^{3}\left|\frac{1}{2}\right\rangle & =\frac{(1-\alpha)}{2}\left|\frac{1}{2}\right\rangle & \hat{L}_{0}\left|\frac{1}{2}\right\rangle=\frac{(1-\alpha)^{2}}{4}\left|\frac{1}{2}\right\rangle .
\end{array}
$$

Thus we conclude that the conformal dimensions of the $\alpha$-twisted representations are $\frac{\alpha^{2}}{4}$, and $\frac{(1-\alpha)^{2}}{4}$, respectively. Since the twisted and untwisted representations are isomorphic as vector spaces, it is straightforward to determine the character of the twisted representation from the untwisted character using (A.26). Because of the free boson realisation of the level one theory, the unspecialised characters $\operatorname{Tr}_{j}\left(q^{L_{0}-c / 24} y^{J_{0}^{3}}\right)$ are

$$
\chi_{0}(q, y)=\frac{1}{\eta(q)} \sum_{n \in \mathbb{Z}} q^{n^{2}} y^{n}, \quad \chi_{\frac{1}{2}}(q, y)=\frac{1}{\eta(q)} \sum_{n \in \mathbb{Z}} q^{\left(n-\frac{1}{2}\right)^{2}} y^{n-\frac{1}{2}}
$$

and hence the corresponding $\alpha$-twisted characters are

$$
\begin{aligned}
& \chi_{0}^{(\alpha)}(q)=\frac{1}{\eta(q)} \sum_{n \in \mathbb{Z}} q^{n^{2}} q^{-n \alpha} q^{\frac{\alpha^{2}}{4}}=\frac{1}{\eta(q)} \sum_{n \in \mathbb{Z}} q^{\frac{(-\alpha+2 n)^{2}}{4}}=\sum_{n \in \mathbb{Z}} \vartheta_{-\alpha+2 n} \\
& \chi_{\frac{1}{2}}^{(\alpha)}(q)=\frac{1}{\eta(q)} \sum_{n \in \mathbb{Z}} q^{\left(n-\frac{1}{2}\right)^{2}} q^{-\alpha\left(n-\frac{1}{2}\right)} q^{\frac{\alpha^{2}}{4}}=\frac{1}{\eta(q)} \sum_{n \in \mathbb{Z}} q^{\frac{(-\alpha+2 n-1)^{2}}{4}}=\sum_{n \in \mathbb{Z}} \vartheta_{-\alpha+2 n-1} .
\end{aligned}
$$

This then matches precisely (3.10). It is also clear from this analysis that the $\mathrm{U}(1)$ charge equals $-\frac{\alpha}{2}+n$ and $-\frac{\alpha}{2}+\left(n-\frac{1}{2}\right)$, respectively, and thus the projection onto the $\mathrm{U}(1)$ singlet states for the left-right spectrum leads precisely to (3.12).

\section{B Identifying twists with weights}

In this appendix we first want to show that the weights $\tilde{\Lambda}$ satisfying $\tilde{\Lambda}_{j} \geq 0$ as well as (5.6) are in one-to-one correspondence with elements in $\hat{\mathbb{T}} / \mathcal{W}$, where $\hat{\mathbb{T}}$ is the Cartan torus of $\mathrm{SU}(N)$, see also [64]. Let $\epsilon_{i}, i=1, \ldots, N$ be the usual orthonormal basis, in terms of which the roots of $\mathfrak{s u}(N)$ are described by

$$
e_{i, j}=\epsilon_{i}-\epsilon_{j}, \quad i \neq j \in\{1, \ldots, N\} .
$$

The simple roots can be taken to be $e_{i} \equiv e_{i, i+1}, i=1, \ldots, N-1$, and the corresponding fundamental weights are

$$
\lambda_{i}=\sum_{j=1}^{i} \epsilon_{j}-\frac{i}{N} \sum_{j=1}^{N} \epsilon_{j}, \quad i=1, \ldots, N-1 .
$$


In this description the Weyl group $\mathcal{W}$ acts by permuting the basis vector $\epsilon_{j}$. Writing $\tilde{\Lambda}$ as

$$
\tilde{\Lambda}=\sum_{s=1}^{N-1} \tilde{\Lambda}_{s} \lambda_{s}=\sum_{i=1}^{N} l_{i} \epsilon_{i}
$$

we have

$$
l_{j}=\sum_{s=j}^{N-1} \tilde{\Lambda}_{s}-\frac{B}{N}, \quad B=\sum_{s=1}^{N-1} s \tilde{\Lambda}_{s} .
$$

By construction we have $\sum_{j} l_{j}=0$. Note further that since all $\tilde{\Lambda}_{s} \geq 0$ it follows that

$$
l_{1} \geq l_{2} \geq \cdots \geq l_{N}
$$

and the condition that $\sum_{s} \tilde{\Lambda}_{s} \leq 1$ becomes

$$
l_{1}-l_{N} \leq 1
$$

Because of the ordering (B.5) this condition is equivalent to $\left|l_{i}-l_{j}\right| \leq 1$ for all $i, j$.

We now want to show that the space of all $\left(l_{1}, \ldots, l_{N}\right)$ satisfying (B.5) and (B.6) is in one-to-one correspondence with elements in $\hat{\mathbb{T}} / \mathcal{W}$. First we recall that the Cartan torus can be identified with the vector space of 'weights'

$$
\alpha=\sum_{j=1}^{N} \alpha_{j} \epsilon_{j} \quad \text { with } \quad \sum_{j=1}^{N} \alpha_{j}=0,
$$

modulo the addition of roots. Because we are only interested in the quotient by the Weyl group, we can use the Weyl group action to order the components, i.e. we may assume without loss of generality that

$$
\alpha_{1} \geq \alpha_{2} \geq \cdots \geq \alpha_{N}
$$

Now there are two cases to consider: if $\Delta \equiv \alpha_{1}-\alpha_{N} \leq 1$, i.e. if all $\left|\alpha_{i}-\alpha_{j}\right| \leq 1$, we identify $\alpha$ directly with $\tilde{\Lambda}$. Alternatively, i.e. if $\Delta \equiv \alpha_{1}-\alpha_{N}>1$, we subtract from $\alpha$ the root $e_{1 N}$, i.e. we consider

$$
\alpha^{\prime}=\alpha-\left(\epsilon_{1}-\epsilon_{N}\right)=\sum_{j=1}^{N} \alpha_{j}^{\prime} \epsilon_{j}=\left(\alpha_{1}-1\right) \epsilon_{1}+\sum_{j=2}^{N-1} \alpha_{j} \epsilon_{j}+\left(\alpha_{N}+1\right) \epsilon_{N} .
$$

Then we reorder (if necessary) the components of $\alpha^{\prime}$ so that they satisfy again (B.8). If the reordering does not involve either $\alpha_{1}^{\prime}$ or $\alpha_{N}^{\prime}$, then $\Delta^{\prime} \leq \Delta-1$ (if either $\alpha_{1}^{\prime}$ or $\alpha_{N}^{\prime}$ is not reordered) or $\Delta^{\prime}=\Delta-2$ (if both are not reordered). On the other hand, if both $\alpha_{1}^{\prime}$ and $\alpha_{N}^{\prime}$ are reordered, then either $\alpha_{1}^{\prime}=\alpha_{N}+1$ or $\alpha_{1}^{\prime}=\alpha_{2} \leq \alpha_{1}$ and either $\alpha_{N}^{\prime}=\alpha_{1}-1$ or $\alpha_{N}^{\prime}=\alpha_{N-1} \geq \alpha_{N}$. In any case it then follows that $\Delta^{\prime} \leq \Delta-$ the most subtle case arises for $\alpha_{1}^{\prime}=\alpha_{N}+1$ and $\alpha_{N}^{\prime}=\alpha_{1}-1$ for which

$$
\Delta^{\prime}=\alpha_{N}+1-\alpha_{1}+1=2-\left(\alpha_{1}-\alpha_{N}\right)<1 .
$$


Continuing in this manner we can thus find a suitable root $e$ so that $\alpha+e$ satisfies $\Delta \leq 1$. (Note that it can happen that in the recursion step the value of $\Delta$ does not decrease, $\Delta^{\prime}=\Delta$, but this is only the case if $\alpha_{1}^{\prime}=\alpha_{2}=\alpha_{1}$ and $\alpha_{N}^{\prime}=\alpha_{N-1}=\alpha_{N}$. It is then clear that at least after $\frac{N}{2}$ iteration steps, the value of $\Delta$ must strictly decrease. Thus the iterative procedure terminates.)

We conclude that any element in $\hat{\mathbb{T}} / \mathcal{W}$ can be brought into a form satisfying (B.5) and (B.6). It is also easy to see (by essentially the same arguments) that not two elements of this form (with the exception of some elements with $\alpha_{1}-\alpha_{N}=1$ ) can differ by a root. This completes the proof of the first statement.

We are actually interested in the Cartan torus $\mathbb{T}$ of $\mathrm{SU}(N) / \mathbb{Z}_{N}$. The generator of the center $\mathbb{Z}_{N}$ can be identified with

$$
c_{N}=\frac{1}{N} \sum_{i=1}^{N-1} i e_{i}=\frac{1}{N} \sum_{i=1}^{N-1} \epsilon_{i}-\frac{(N-1)}{N} \epsilon_{N} .
$$

The Cartan torus $\mathbb{T}$ is thus obtained from $\hat{\mathbb{T}}$ upon dividing out the multiples of $c_{N}$, and the quotient space $\mathbb{T} / \mathcal{W}$ is obtained from $\hat{\mathbb{T}} / \mathcal{W}$ by dividing out the lattice that is generated by the vectors

$$
c_{j}=\frac{1}{N} \sum_{i \neq j} \epsilon_{i}-\frac{(N-1)}{N} \epsilon_{j}, \quad j=1, \ldots, N,
$$

i.e. by the image vectors of $c_{N}$ under the Weyl group action. In the quotient space $\mathbb{T} / \mathcal{W}$ we can therefore reduce the vectors $\alpha$ further to those that satisfy in addition

$$
\alpha_{j}-\alpha_{j+1} \leq \frac{1}{2} \quad \text { for all } \quad j=1, \ldots, N-1
$$

as well as

$$
\alpha_{1}-\alpha_{N} \leq 1-\max _{i}\left(\alpha_{i}-\alpha_{i+1}\right) .
$$

In order to see that (B.13) can be achieved, suppose that $\alpha_{j}-\alpha_{j+1}>\frac{1}{2}$ for some $1 \leq j \leq$ $N-1$. (Since $\alpha_{1}-\alpha_{N} \leq 1$, this can happen at most for one $j$.) Then it follows that

$$
\alpha^{\prime}=\alpha+\sum_{i=1}^{j} c_{i}
$$

after reordering has the form

$$
\alpha^{\prime}=\left(\alpha_{j+1}+\frac{j}{N}, \ldots, \alpha_{N}+\frac{j}{N}, \alpha_{1}-1+\frac{j}{N}, \ldots, \alpha_{j}-1+\frac{j}{N}\right) .
$$

Since $\alpha_{1}^{\prime}-\alpha_{N}^{\prime}=1-\left(\alpha_{j}-\alpha_{j+1}\right)<\frac{1}{2}<1$, the vector $\alpha^{\prime}$ satisfies then condition (B.13), as well as (B.6).

In order to see that we can in addition impose (B.14), let $j$ be the value for which $\alpha_{j}-\alpha_{j+1}$ is maximal. If $\alpha_{1}-\alpha_{N}>1-\left(\alpha_{j}-\alpha_{j+1}\right)$, we consider $\alpha^{\prime}=\alpha+\sum_{i=1}^{j} c_{j}$ of the form (B.16). Then the differences $\alpha_{i}^{\prime}-\alpha_{i+1}^{\prime}$ for $i \neq N-j$ agree with the differences $\alpha_{l}-\alpha_{l+1}$ with $l \neq j$, while for $i=N-j$ we now have

$$
\alpha_{N-j}^{\prime}-\alpha_{N-j+1}^{\prime}=\alpha_{N}+\frac{j}{N}-\left(\alpha_{1}-1+\frac{j}{N}\right)=1-\left(\alpha_{1}-\alpha_{N}\right)<\alpha_{j}-\alpha_{j+1} \leq \frac{1}{2}
$$


since $\left(\alpha_{1}-\alpha_{N}\right)>1-\left(\alpha_{j}-\alpha_{j+1}\right) \geq \frac{1}{2}$. Because all the differences $\alpha_{i}^{\prime}-\alpha_{i+1}^{\prime}$ are smaller or equal than $\alpha_{j}-\alpha_{j+1}$, the overall difference $\alpha_{1}^{\prime}-\alpha_{N}^{\prime}$ now satisfies the condition (B.14)

$$
\alpha_{1}^{\prime}-\alpha_{N}^{\prime}=1-\left(\alpha_{j}-\alpha_{j+1}\right) \leq 1-\max _{i}\left(\alpha_{i}^{\prime}-\alpha_{i+1}^{\prime}\right)
$$

We close by noting that the allowed non-trivial weights of the level one algebra are of the form $\Lambda_{i}^{(j)}=\delta_{i j}$ for $j=1, \ldots, N-1$, and hence equal in the orthogonal basis

$$
\Lambda^{(j)}=-\sum_{i=1}^{j} c_{j} .
$$

It is then manifest from the above discussion that $\hat{\mathbb{T}} / \mathcal{W}$ can be written as the union of $\mathbb{T} / \mathcal{W}$, together with the shifted weights $\Lambda^{(j)}+\mathbb{T} / \mathcal{W}$. The latter weights appear in the twisted version of the level one $\Lambda^{(j)}$ representation (where we twist again by an element in $\mathbb{T} / \mathcal{W}$ ). This mirrors precisely what happened for $N=2$, compare eq. (A.35).

Open Access. This article is distributed under the terms of the Creative Commons Attribution License which permits any use, distribution and reproduction in any medium, provided the original author(s) and source are credited.

\section{References}

[1] B. Sundborg, Stringy gravity, interacting tensionless strings and massless higher spins, Nucl. Phys. Proc. Suppl. 102 (2001) 113 [InSPIRE].

[2] E. Witten, Spacetime reconstrution, talk at the John Schwarz $60^{\text {th }}$ birthday symposium, http://theory.caltech.edu/jhs60/witten/1.html.

[3] A. Mikhailov, Notes on higher spin symmetries, hep-th/0201019 [INSPIRE].

[4] E. Sezgin and P. Sundell, Massless higher spins and holography, Nucl. Phys. B 644 (2002) 303 [Erratum ibid. B 660 (2003) 403] [hep-th/0205131] [INSPIRE].

[5] M. Vasiliev, Nonlinear equations for symmetric massless higher spin fields in (A)dS(d), Phys. Lett. B 567 (2003) 139 [hep-th/0304049] [INSPIRE].

[6] M.A. Vasiliev, Higher spin gauge theories: star product and AdS space, in The many faces of the superworld, Y. Golfand and M.A. Shifman eds., World Scientific, Singapore (1999), hep-th/9910096 [INSPIRE].

[7] X. Bekaert, S. Cnockaert, C. Iazeolla and M. Vasiliev, Nonlinear higher spin theories in various dimensions, hep-th/0503128 [INSPIRE].

[8] C. Iazeolla, On the algebraic structure of higher-spin field equations and new exact solutions, arXiv:0807.0406 [INSPIRE].

[9] A. Campoleoni, Metric-like lagrangian formulations for higher-spin fields of mixed symmetry, Riv. Nuovo Cim. 033 (2010) 123 [arXiv:0910.3155] [INSPIRE].

[10] I. Klebanov and A. Polyakov, AdS dual of the critical $O(N)$ vector model, Phys. Lett. B 550 (2002) 213 [hep-th/0210114] [inSPIRE]. 
[11] E. Sezgin and P. Sundell, Holography in $4 D$ (super) higher spin theories and a test via cubic scalar couplings, JHEP 07 (2005) 044 [hep-th/0305040] [INSPIRE].

[12] S. Giombi and X. Yin, Higher spin gauge theory and holography: the three-point functions, JHEP 09 (2010) 115 [arXiv:0912.3462] [INSPIRE].

[13] S. Giombi and X. Yin, Higher spins in AdS and twistorial holography, JHEP 04 (2011) 086 [arXiv: 1004.3736] [INSPIRE].

[14] S. Giombi and X. Yin, On higher spin gauge theory and the critical $O(N)$ model, arXiv:1105.4011 [INSPIRE].

[15] R. de Mello Koch, A. Jevicki, K. Jin and J.P. Rodrigues, $A d S_{4} / C F T_{3}$ construction from collective fields, Phys. Rev. D 83 (2011) 025006 [arXiv: 1008.0633] [inSPIRE].

[16] M.R. Douglas, L. Mazzucato and S.S. Razamat, Holographic dual of free field theory, Phys. Rev. D 83 (2011) 071701 [arXiv:1011.4926] [INSPIRE].

[17] S.H. Shenker and X. Yin, Vector models in the singlet sector at finite temperature, arXiv:1109.3519 [INSPIRE].

[18] O. Aharony, G. Gur-Ari and R. Yacoby, $D=3$ bosonic vector models coupled to Chern-Simons gauge theories, JHEP 03 (2012) 037 [arXiv:1110.4382] [INSPIRE].

[19] S. Giombi et al., Chern-Simons theory with vector fermion matter, arXiv:1110.4386 [INSPIRE].

[20] J. Maldacena and A. Zhiboedov, Constraining conformal field theories with a higher spin symmetry, arXiv:1112.1016 [INSPIRE].

[21] M.R. Gaberdiel and R. Gopakumar, An $A d S_{3}$ dual for minimal model CFTs, Phys. Rev. D 83 (2011) 066007 [arXiv: 1011.2986] [InSPIRE].

[22] S. Prokushkin and M.A. Vasiliev, Higher spin gauge interactions for massive matter fields in 3D AdS space-time, Nucl. Phys. B 545 (1999) 385 [hep-th/9806236] [INSPIRE].

[23] S. Prokushkin and M.A. Vasiliev, 3D higher spin gauge theories with matter, hep-th/9812242 [INSPIRE].

[24] P. Bouwknegt and K. Schoutens, $W$ symmetry in conformal field theory, Phys. Rept. 223 (1993) 183 [hep-th/9210010] [InSPIRE].

[25] M. Henneaux and S.-J. Rey, Nonlinear $W_{\infty}$ as asymptotic symmetry of three-dimensional higher spin Anti-de Sitter gravity, JHEP 12 (2010) 007 [arXiv: 1008.4579] [InSPIRE].

[26] A. Campoleoni, S. Fredenhagen, S. Pfenninger and S. Theisen, Asymptotic symmetries of three-dimensional gravity coupled to higher-spin fields, JHEP 11 (2010) 007 [arXiv: 1008.4744] [INSPIRE].

[27] J. Brown and M. Henneaux, Central charges in the canonical realization of asymptotic symmetries: an example from three-dimensional gravity, Commun. Math. Phys. 104 (1986) 207 [InSPIRE].

[28] M.R. Gaberdiel and T. Hartman, Symmetries of holographic minimal models, JHEP 05 (2011) 031 [arXiv:1101.2910] [INSPIRE].

[29] A. Campoleoni, S. Fredenhagen and S. Pfenninger, Asymptotic W-symmetries in three-dimensional higher-spin gauge theories, JHEP 09 (2011) 113 [arXiv:1107.0290] [INSPIRE]. 
[30] M.R. Gaberdiel, R. Gopakumar, T. Hartman and S. Raju, Partition functions of holographic minimal models, JHEP 08 (2011) 077 [arXiv: 1106.1897] [INSPIRE].

[31] M.R. Gaberdiel, R. Gopakumar and A. Saha, Quantum $W$-symmetry in $A d S_{3}$, JHEP 02 (2011) 004 [arXiv: 1009.6087] [INSPIRE].

[32] C.-M. Chang and X. Yin, Higher spin gravity with matter in $A d S_{3}$ and its CFT dual, arXiv:1106.2580 [INSPIRE].

[33] C. Ahn, The coset spin-4 Casimir operator and its three-point functions with scalars, JHEP 02 (2012) 027 [arXiv:1111.0091] [INSPIRE].

[34] M. Ammon, P. Kraus and E. Perlmutter, Scalar fields and three-point functions in D=3 higher spin gravity, arXiv:1111.3926 [INSPIRE].

[35] K. Papadodimas and S. Raju, Correlation functions in holographic minimal models, Nucl. Phys. B 856 (2012) 607 [arXiv:1108.3077] [INSPIRE].

[36] C. Ahn, The large- $N$ 't Hooft limit of coset minimal models, JHEP 10 (2011) 125 [arXiv:1106.0351] [INSPIRE].

[37] M.R. Gaberdiel and C. Vollenweider, Minimal model holography for $\mathrm{SO}(2 \mathrm{~N})$, JHEP 08 (2011) 104 [arXiv:1106.2634] [INSPIRE].

[38] M. Gutperle and P. Kraus, Higher spin black holes, JHEP 05 (2011) 022 [arXiv:1103.4304] [INSPIRE].

[39] M. Ammon, M. Gutperle, P. Kraus and E. Perlmutter, Spacetime geometry in higher spin gravity, JHEP 10 (2011) 053 [arXiv:1106.4788] [INSPIRE].

[40] P. Kraus and E. Perlmutter, Partition functions of higher spin black holes and their CFT duals, JHEP 11 (2011) 061 [arXiv:1108.2567] [INSPIRE].

[41] D. Roggenkamp and K. Wendland, Limits and degenerations of unitary conformal field theories, Commun. Math. Phys. 251 (2004) 589 [hep-th/0308143] [INSPIRE].

[42] I. Runkel and G. Watts, A nonrational CFT with $c=1$ as a limit of minimal models, JHEP 09 (2001) 006 [hep-th/0107118] [INSPIRE].

[43] S. Fredenhagen and V. Schomerus, Boundary Liouville theory at $c=1$, JHEP 05 (2005) 025 [hep-th/0409256] [INSPIRE].

[44] V. Schomerus, Rolling tachyons from Liouville theory, JHEP 11 (2003) 043 [hep-th/0306026] [INSPIRE].

[45] R. Howe, Remarks on classical invariant theory, Trans. Amer. Math. Soc. 313 (1989) 539.

[46] L.J. Dixon, J.A. Harvey, C. Vafa and E. Witten, Strings on orbifolds, Nucl. Phys. B 261 (1985) 678 [inSPIRE].

[47] L.J. Dixon, J.A. Harvey, C. Vafa and E. Witten, Strings on orbifolds. 2, Nucl. Phys. B 274 (1986) 285 [inSPIRE].

[48] K. Graham, I. Runkel and G. Watts, Minimal model boundary flows and $c=1$ CFT, Nucl. Phys. B 608 (2001) 527 [hep-th/0101187] [INSPIRE].

[49] S. Hamidi and C. Vafa, Interactions on orbifolds, Nucl. Phys. B 279 (1987) 465 [inSPIRE].

[50] L.J. Dixon, D. Friedan, E.J. Martinec and S.H. Shenker, The conformal field theory of orbifolds, Nucl. Phys. B 282 (1987) 13 [INSPIRE]. 
[51] R. Dijkgraaf, C. Vafa, E.P. Verlinde and H.L. Verlinde, The operator algebra of orbifold models, Commun. Math. Phys. 123 (1989) 485 [INSPIRE].

[52] M. Gaberdiel, A. Recknagel and G. Watts, The conformal boundary states for $\mathrm{SU}(2)$ at level 1, Nucl. Phys. B 626 (2002) 344 [hep-th/0108102] [INSPIRE].

[53] A.Y. Alekseev and V. Schomerus, D-branes in the WZW model, Phys. Rev. D 60 (1999) 061901 [hep-th/9812193] [INSPIRE].

[54] M.R. Douglas and G.W. Moore, D-branes, quivers and ALE instantons, hep-th/9603167 [INSPIRE].

[55] M.R. Douglas, D-branes and discrete torsion, hep-th/9807235 [INSPIRE].

[56] M.R. Douglas and B. Fiol, D-branes and discrete torsion. 2, JHEP 09 (2005) 053 [hep-th/9903031] [INSPIRE].

[57] M.R. Gaberdiel, Discrete torsion orbifolds and D-branes, JHEP 11 (2000) 026 [hep-th/0008230] [INSPIRE].

[58] S. Fredenhagen, Boundary conditions in Toda theories and minimal models, JHEP 02 (2011) 052 [arXiv: 1012.0485] [INSPIRE].

[59] M.R. Gaberdiel, Abelian duality in WZW models, Nucl. Phys. B 471 (1996) 217 [hep-th/9601016] [INSPIRE].

[60] S. Fredenhagen and D. Wellig, A common limit of super Liouville theory and minimal models, JHEP 09 (2007) 098 [arXiv:0706.1650] [INSPIRE].

[61] A. Castro, R. Gopakumar, M. Gutperle and J. Raeymaekers, Conical defects in higher spin theories, JHEP 02 (2012) 096 [arXiv:1111.3381] [INSPIRE].

[62] J.R. David, M.R. Gaberdiel and R. Gopakumar, The heat kernel on $A d S_{3}$ and its applications, JHEP 04 (2010) 125 [arXiv:0911.5085] [INSPIRE].

[63] P. Goddard and D.I. Olive, Kac-Moody and Virasoro algebras in relation to quantum physics, Int. J. Mod. Phys. A 1 (1986) 303 [INSPIRE].

[64] J.J. Duistermaat and J.A.C. Kolk, Lie groups, Springer, U.S.A. (2000). 